\title{
Validation Study of Unnotched Charpy and Taylor-Anvil Impact Experiments Using Kayenta
}

Krishna Kamojjala, Jeffrey Lacy

Henry Chu

Rebecca Brannon

December 2014

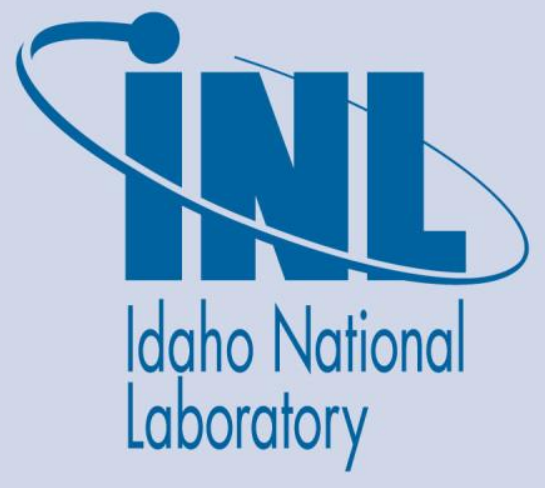

The INL is a U.S. Department of Energy National Laboratory operated by Battelle Energy Alliance 


\section{DISCLAIMER}

This information was prepared as an account of work sponsored by an agency of the U.S. Government. Neither the U.S. Government nor any agency thereof, nor any of their employees, makes any warranty, expressed or implied, or assumes any legal liability or responsibility for the accuracy, completeness, or usefulness, of any information, apparatus, product, or process disclosed, or represents that its use would not infringe privately owned rights. References herein to any specific commercial product, process, or service by trade name, trade mark, manufacturer, or otherwise, does not necessarily constitute or imply its endorsement, recommendation, or favoring by the U.S. Government or any agency thereof. The views and opinions of authors expressed herein do not necessarily state or reflect those of the U.S. Government or any agency thereof. 
INL/EXT-14-33981

Revision 0

\title{
Validation Study of Unnotched Charpy and Taylor-Anvil Impact Experiments using Kayenta
}

\author{
Krishna Kamojjala \\ Jeffrey Lacy \\ Henry Chu \\ Rebecca Brannon
}

December 2014

Idaho National Laboratory

Specific Manufacturing Capability

Idaho Falls, Idaho 83415 


\begin{abstract}
Validation of a single computational model with multiple available strain-tofailure fracture theories is presented through experimental tests and numerical simulations of the standardized unnotched Charpy and Taylor-anvil impact tests, both run using the same material model (Kayenta). Unnotched Charpy tests are performed on rolled homogeneous armor steel. The fracture patterns using Kayenta's various failure options that include aleatory uncertainty and scale effects are compared against the experiments. Other quantities of interest include the average value of the absorbed energy and bend angle of the specimen.

Taylor-anvil impact tests are performed on Ti6Al4V titanium alloy. The impact speeds of the specimen are $321 \mathrm{~m} / \mathrm{s}$ and $393 \mathrm{~m} / \mathrm{s}$. The goal of the numerical work is to reproduce the damage patterns observed in the laboratory. For the numerical study, the Johnson-Cook failure model [1] is used as the ductile fracture criterion, and aleatory uncertainty is applied to rate-dependence parameters to explore its effect on the fracture patterns.
\end{abstract}




\section{CONTENTS}

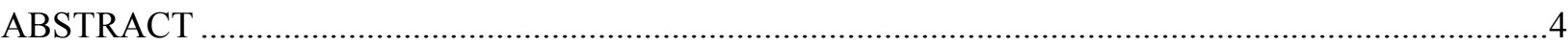

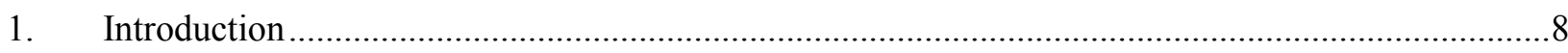

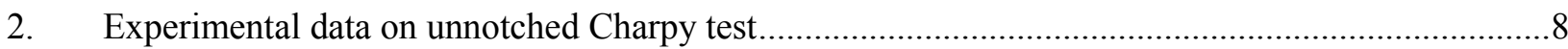

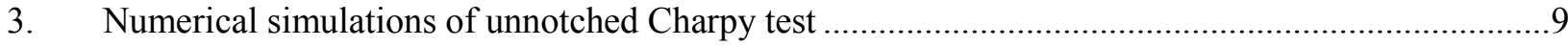

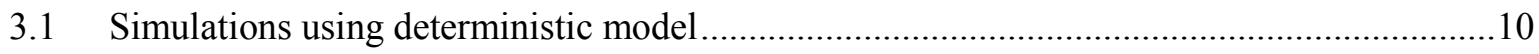

3.2 Simulations using statistical variability of strength and scale effects ...................................11

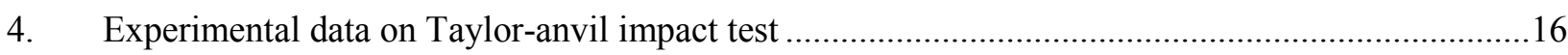

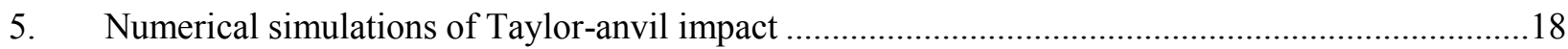

5.1 Verification of rate dependence, hardening and softening in Kayenta ................................18

5.1.1 Strain-to-failure softening without rate dependence.............................................19

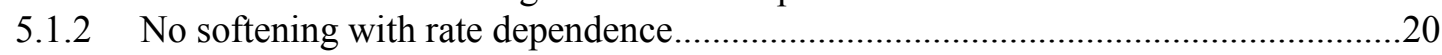

5.1.3 Strain-to-failure softening with rate dependence..................................................23

5.1.4 Strain-to-failure softening with rate dependence and hardening ...............................24

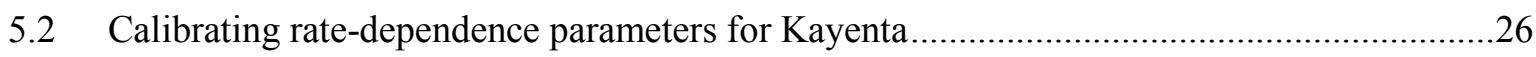

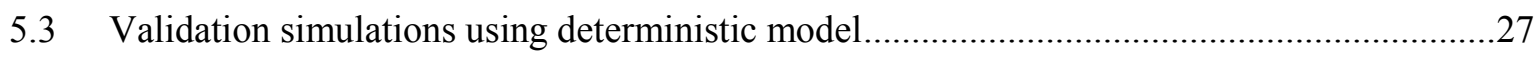

5.4 Validation simulations using variability applied to rate-dependence parameters.....................30

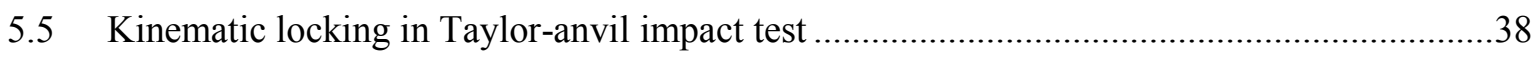

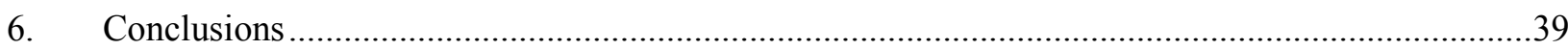

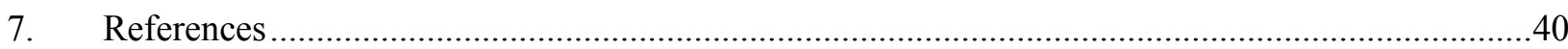

\section{FIGURES}

Figure 1: Experimental observations of damage patterns.

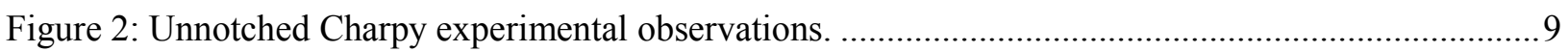

Figure 3: Convergence study using 2D plane-strain simulations...................................................... 10

Figure 4: Damage in 3D simulations using different softening options. ..................................................11

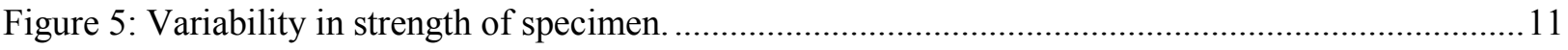

Figure 6: The same simulations as in Figure 3 except run using Kayenta's option for statistical variability and scale effects in strength.

Figure 7: Plots of damage (red is damaged, and blue is intact material) using constant strain-tofailure softening model in Kayenta.

Figure 8: Plots of damage (red is damaged, and blue is intact material) using constant strain-tofailure softening model in Kayenta.

Figure 9: Plots of damage (red is damaged material, and blue is intact material) using JohnsonCook softening model in Kayenta.

Figure 10: Plots of damage (red is damaged material, and blue is intact material) using constant strain-to-failure softening with an artificially increased striker speed of $51.47 \mathrm{~m} / \mathrm{s}$.

Figure 11: Plots of damage (red is damaged material, and blue is intact material) using constant strain-to-failure softening model with striker speed of $5.147 \mathrm{~m} / \mathrm{s}$. 
Figure 12: Time history of the K.E. of the striker for three meshes using statistical variability in strength with no scale effects.

Figure 13: Time history of the K.E. of the striker for three meshes using statistical variability in strength and scale effects.

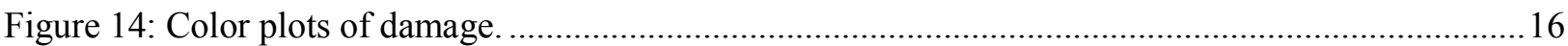

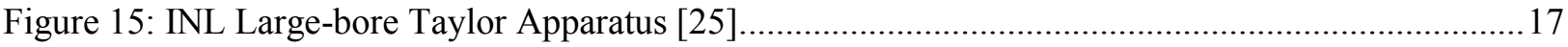

Figure 16: Experimental observations of damage patterns at specimen speed of $321 \mathrm{~m} / \mathrm{s}$....................... 17

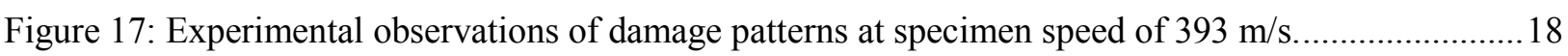

Figure 18: Normalized time history of the parameter COHER at low strain rates (softening only)..........19

Figure 19: Normalized stress difference vs. normalized strain at low strain rates (softening only)...........19

Figure 20: Normalized time history of the parameter COHER at high strain rates (softening only).

Figure 21: Normalized stress difference vs. normalized strain at high strain rates (softening only).........20

Figure 22: Normalized time history of the parameter COHER at low strain rates (rate dependence only)

Figure 23: Normalized stress difference vs. normalized strain at low strain rates (rate dependence only).

Figure 24: Normalized time history of the parameter COHER at high strain rates (Rate dependence only).

Figure 25: Normalized stress difference vs. normalized strain at high strain rates (rate dependence only).

Figure 26: Normalized time history of the COHER at low strain rates (both rate dependence and strain-to-failure softening).

Figure 27: Normalized stress difference vs. normalized strain at low strain rates (both rate dependence and strain-to-failure softening).

Figure 28: Normalized time history of the parameter COHER at high strain rates (both rate dependence and strain-to-failure softening).

Figure 29: Normalized stress difference vs. normalized strain at high strain rates (both rate dependence and strain-to-failure softening).

Figure 30: Normalized time history of the COHER at high strain rates (rate dependence, hardening and softening).

Figure 31: Normalized stress difference vs. normalized strain at high strain rates (rate dependence, hardening and softening).

Figure 32: Trend tests on Taylor-anvil impact simulations using deterministic material properties (i.e., no statistical variability or scale effects).

Figure 33: $\tau$ as a function of strain rate.

Figure 34: Plot of damage using Johnson-Cook failure model without thermal effects. ..........................28

Figure 35: Plot of damage using Johnson-Cook failure model without thermal effects. ..........................28

Figure 36: Plot of damage using Johnson-Cook failure model with thermal effects.................................29

Figure 37: Plot of damage using Johnson-Cook failure model with thermal effects................................29

Figure 38: Severe mesh dependency for damage using a deterministic scale-insensitive model...............30 
Figure 39: Least squares fit of Ti6A14V experimental data [35] to Eq. (3)................................................. 31

Figure 40: Sensitivity of rate-dependence parameter T1 on damage without EOS..................................32

Figure 41: Sensitivity of rate-dependence parameter T1 on damage with EOS........................................33

Figure 42: Sensitivity of rate-dependence parameter T1 on damage without EOS...................................34

Figure 43: Sensitivity of rate-dependence parameter T1 on damage with EOS........................................34

Figure 44: Color plot of damage with statistics put on T2. Blue is intact material and red is

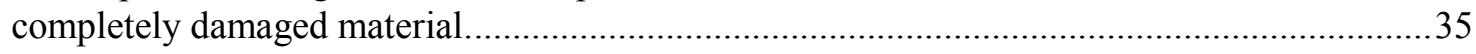

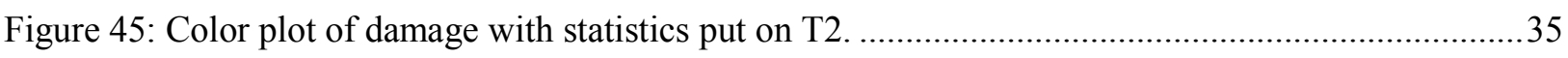

Figure 46: Color plot of damage and equivalent plastic strain with statistics put on T2 ..........................36

Figure 47: Same simulation as Figure 46 except run with particles conforming with the boundary.........36

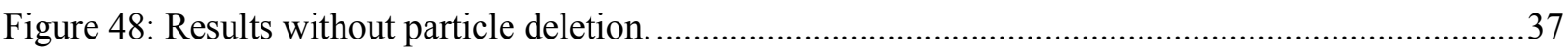

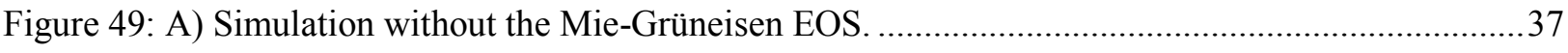

Figure 50: Effect of variability on melt temperature and Johnson-Cook failure parameter D5 on

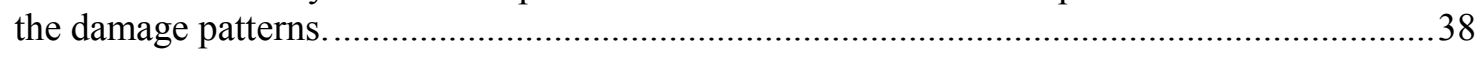

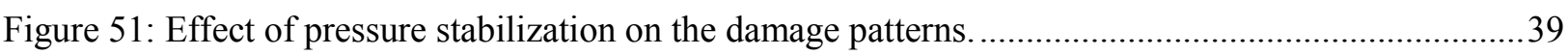

\section{TABLES}

Table 1: Mesh configurations and the amount of energy absorbed using statistical variability in

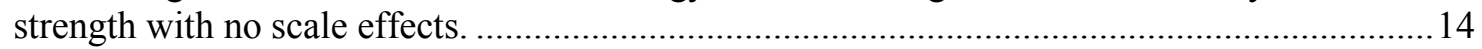

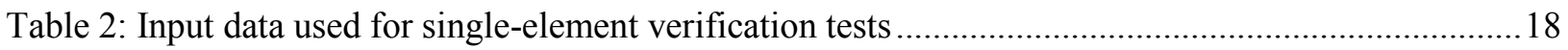

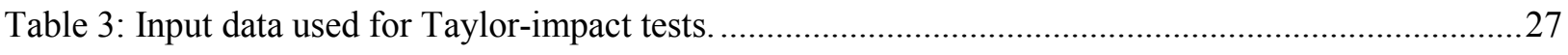

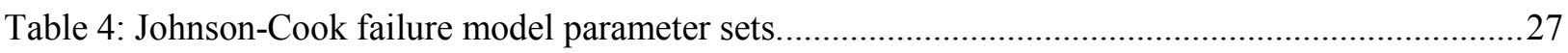

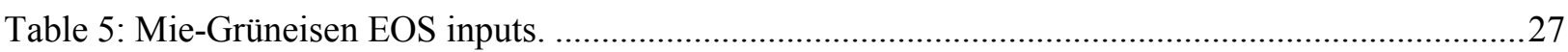




\section{Introduction}

Applications in defense, aerospace, etc. use high-strength metals and alloys that can resist penetration and damage. Rolled homogeneous armor (RHA) steel is one of the most commonly used materials in armored vehicles and ammunition testing because of its low cost and structural efficiency (cf. [2]). Titanium alloys are primarily used in aerospace applications (cf. [3]) because of their high strength at extreme temperatures and immunity to corrosion. Predictive simulations of damage in such high-strength materials are of ongoing interest for the scientific community. Several strain-to-failure models, including void-growth models [4], [5], [6] and conventional damage models [7], [1], [8] have been proposed in the literature to quantify damage. In void-growth models, damage is assumed to initiate when the void volume reaches a critical value. Generally, in conventional damage models, a scalar damage variable increase from zero to unity (fully damaged state) under plastic loading, and these models assume no coupling between plasticity and damage. The disadvantage of these conventional models is that there is no loss of strength or stiffness until the scalar damage variable reaches unity. In Kayenta, softening is related to material strength, and the material progressively loses stiffness and load carrying capacity when it undergoes irreversible damage. The rate of damage progression can be controlled so that Kayenta can emulate a broad range of other conventional damage models, which facilitates deciding which of them (if any) appears to be imposing desirable model behaviors. This chapter provides a systematic validation study on damage for the unnotched Charpy test (performed on RHA steel) and the Taylor-anvil impact test (performed on Ti6Al4V alloy) using Kayenta. Sections 2 and 3 provide the experimental details and the numerical study performed on the unnotched Charpy test. And the subsequent Sections 4 and 5 provide the details on the Taylor-anvil impact tests.

The Charpy test [9] is a standardized test used to determine impact toughness, which is quantified by the amount of energy absorbed by the specimen before fracture. The specimen is supported against two anvils, and a pendulum with a striker is released to impact the specimen. For validating the unnotched Charpy test, the quantities of interest for comparison are the bend angle of the specimen and the amount of energy absorbed by the specimen. As per ASTM E23 [10] standard, "the absorbed energy is taken as the difference between the energy in the striking member at the instant of impact with the specimen, and the energy remained after breaking the specimen." The Taylor-anvil impact test on the other hand, is used to determine the dynamic behavior of materials at high strain rates. Ref [11] first impacted a cylindrical specimen on a flat rigid target to determine the dynamic yield stress. Countless papers about the Tayloranvil impact test may be found in the literature. Most of the experimental [12], [13], and numerical [14] and [15] work for the Taylor-anvil impact test focused on determining the constitutive model coefficients and dynamic yield stresses, but relatively few papers presented the numerical predictions of fracture mechanisms [16] and [17] observed in the Taylor-anvil impact tests. This chapter provides a validation study aimed at assessing which features in prevailing damage and plasticity models appear to be important to reproduce fracture patterns observed in the laboratory for Taylor-anvil impact tests on Ti6Al4V.

\section{Experimental data on unnotched Charpy test}

Six unnotched Charpy experiments were conducted on RHA steel at Idaho National Laboratory. The RHA specimens were tested in a machine meeting the requirements of ASTM E23 [10]. The pendulum was a C-type, with anvil and striker geometry conforming to that of the standard [10]. The impactor mass was $60 \mathrm{lb}$. $(27.26 \mathrm{~kg})$. Given the arm radius, the striker velocity was calculated to be $5.147 \mathrm{~m} / \mathrm{s}$. All tests were conducted with the same procedure (no striker or velocity changes). Striker kinetic energy was on the order of $361 \mathrm{~J}(266 \mathrm{ft} . / \mathrm{lb}$.), and the specimen dimensions were $5 \times 10 \times 55 \mathrm{~mm}$. The average value of the absorbed energy, and bend angle in these six experiments was taken as the measure for comparison of the experimental data and numerical simulations. Figure 1 shows the deformed specimens with the damage patterns at the bottom (convex side) of the specimen. Large plastic deformation with multiple cracks on the tension surface was observed. The average amount of energy absorbed in Figure 2 was $106.833 \mathrm{ft} . / \mathrm{lb}$. (144.84 Joules), and the average bend angle was $95^{\circ}$. The definition used to calculate the amount of energy absorbed in the specimen from numerical simulations was Initial K.E. of the striker - Final K.E. of the striker where K.E. is the kinetic energy. This is likely to be the upper bound on the inelastic absorbed 
energy since it does not remove reversible elastic stored energy. Bending angle definition was also taken from Ref [10].
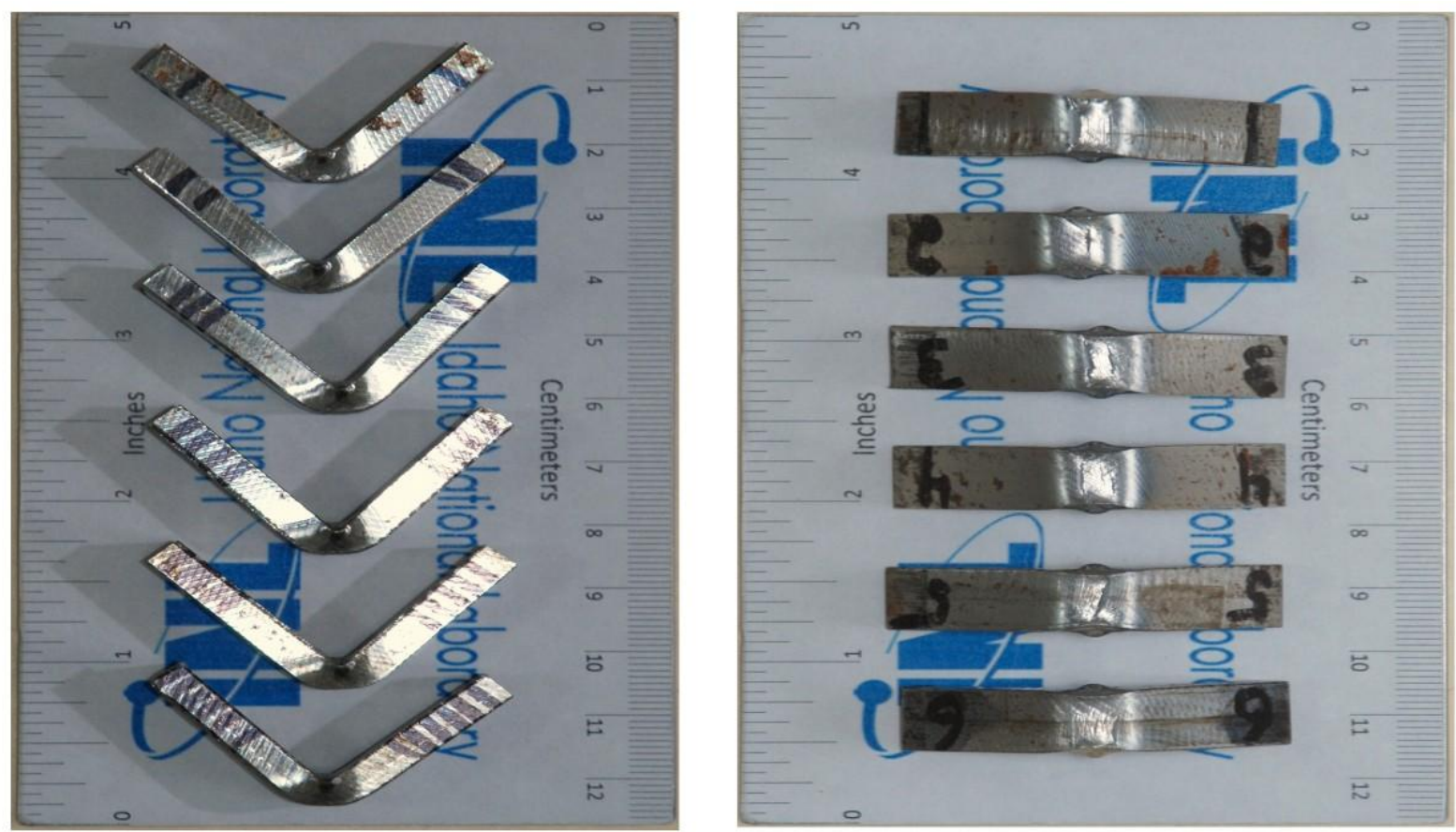

Figure 1: Experimental observations of damage patterns.

\begin{tabular}{|c|c|c|c|c|c|c|c|}
\hline Date & Sample ID & Length & Width & Thickness & Orientation & $\begin{array}{c}\text { Absorbed } \\
\text { Energy }\end{array}$ & Bend Angle \\
\hline$\# 1$ & RHA & 2.011 & .392 & .197 & N/A & 99.3 & 92 \\
\hline & & & & & & & \\
\hline$\# 2$ & RHA & 2.163 & .392 & .197 & N/A & 110.7 & 95 \\
\hline & & & & & & & \\
\hline$\# 3$ & RHA & 2.166 & .391 & .196 & N/A & 107.9 & 97 \\
\hline & & & & & & & \\
\hline$\# 4$ & RHA & 2.170 & .394 & .197 & N/A & 112.0 & 98 \\
\hline & & & & & & & \\
\hline$\# 5$ & RHA & 2.155 & .393 & .197 & N/A & 108.3 & 96 \\
\hline & & & & & & & \\
\hline$\# 6$ & RHA & 2.044 & .392 & .197 & N/A & 102.8 & 92 \\
\hline & & & & & & & \\
\hline
\end{tabular}

Figure 2: Unnotched Charpy experimental observations. Lengths are in inches, energy is in ft./lb., and the bend angle is in degrees.

\section{Numerical simulations of unnotched Charpy test}

All of the numerical simulations described herein use the open-source Uintah MPM framework [18] and Kayenta [19] as the constitutive model. In classical Kayenta softening routines, TFAIL and TGROW are the two parameters that control the softening. TFAIL is the material-specific characteristic failure time for a given loading condition and element size. When using the strain-to-failure option in Kayenta, TFAIL is set to an appropriate, dynamically adjusted value that would produce failure at the designated strain if the strain rate calculated in the problem were to be held constant. A numerical study was performed on the unnotched Charpy test using the following softening options available in Kayenta:

- Time-to-failure

- Constant strain-to-failure

- Johnson-Cook failure 
2D and 3D damage simulations are presented in Section 3.1 using the deterministic scale- insensitive model, where lack of convergence is evident. Section 3.2 shows improved results when using statistical variability in strength and scale effects.

\subsection{Simulations using deterministic model}

Figure 3 shows the convergence study on damage for 2D plane-strain simulations using the different softening options in Kayenta. Mesh resolution increases from left to right. Red indicates damage and blue indicates intact material. For all softening options, the damage zone does not converge with mesh refinement. Since these simulations were symmetric about the vertical axis, the results using this deterministic option should (and do) exhibit the same symmetry. Comparing these 2D simulations against the experiments, the Johnson-cook failure option was under-predicting damage in the specimen, and the time-to-failure option was over-predicting damage in the specimen. For the constant strain-to-failure softening option, the strain-to-failure was chosen to be 0.3 .
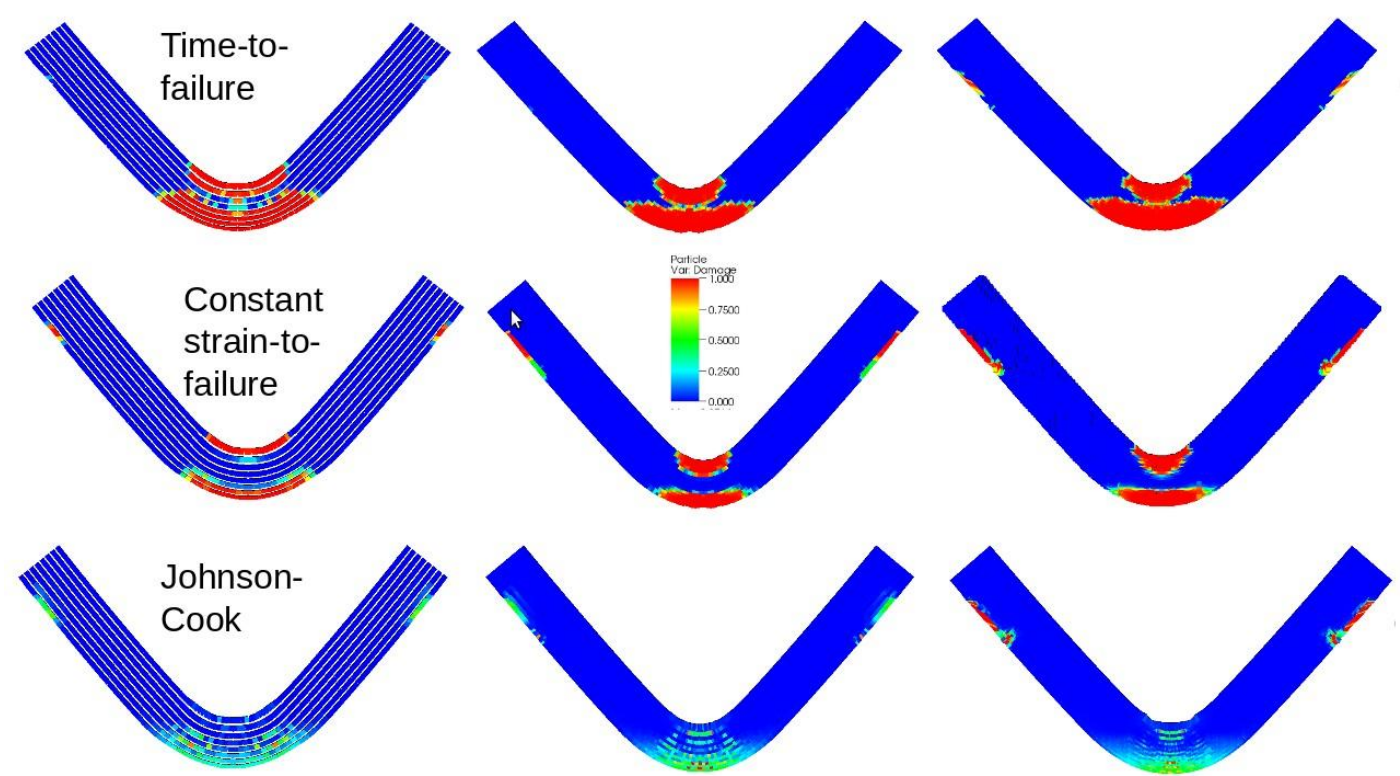

Figure 3: Convergence study using 2D plane-strain simulations. Mesh resolution increases from left to right. Red indicates damaged and blue indicates intact material.

When the same simulations were run in 3D (Figure 4), similar predictions to the 2D simulations were observed with the time-to-failure softening option over-predicting the damage and Johnson-Cook softening under-predicting the damage compared to the experiments. The prediction using a constant strain-to-failure softening option was closer to the experiments, but convergence issues persisted. Brannon [20] considerably reduced mesh sensitivity in axisymmetric dynamic sphere-indentation problems using statistical variability of strength and scale effects. The same features were therefore explored on unnotched Charpy simulations, which are detailed in the next section. 

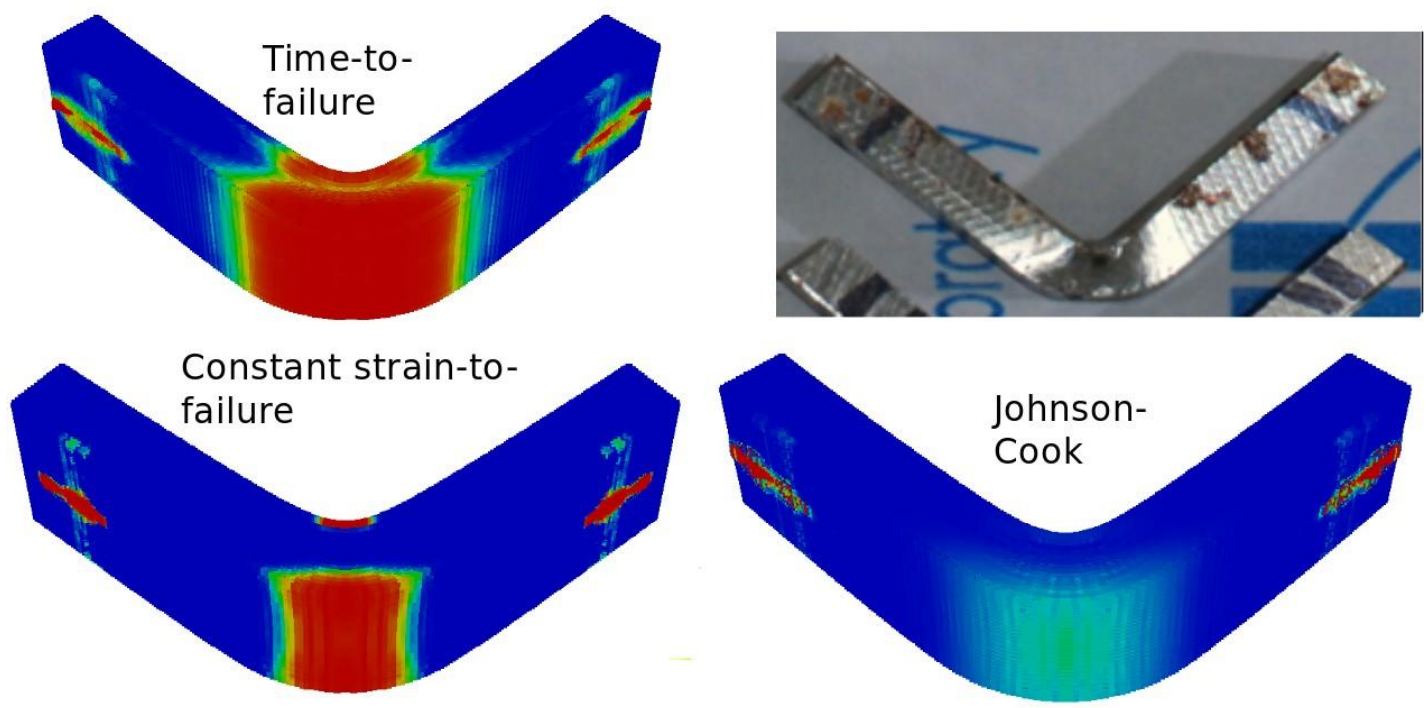

Figure 4: Damage in 3D simulations using different softening options. Red indicates damaged and blue indicates intact material.

\subsection{Simulations using statistical variability of strength and scale effects}

Verification of the implementation of statistics in strength was performed using trend tests by running $2 \mathrm{D}$ plane-strain simulations of the unnotched Charpy test.

Figure 5 shows the variable strength assigned to each particle. The simulations in the top row of Figure 3 were re-run using statistical variability in strength. Since these simulations were run with variability, as expected, the results predicted the loss of symmetry which can be seen in Figure 6. Once the implementation of statistics in strength was verified using these trend tests, 3D simulations were run using this feature.

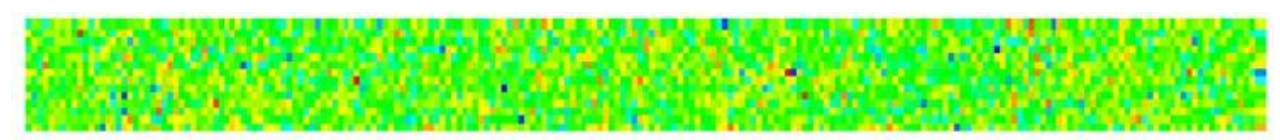

Figure 5: Variability in strength of specimen.

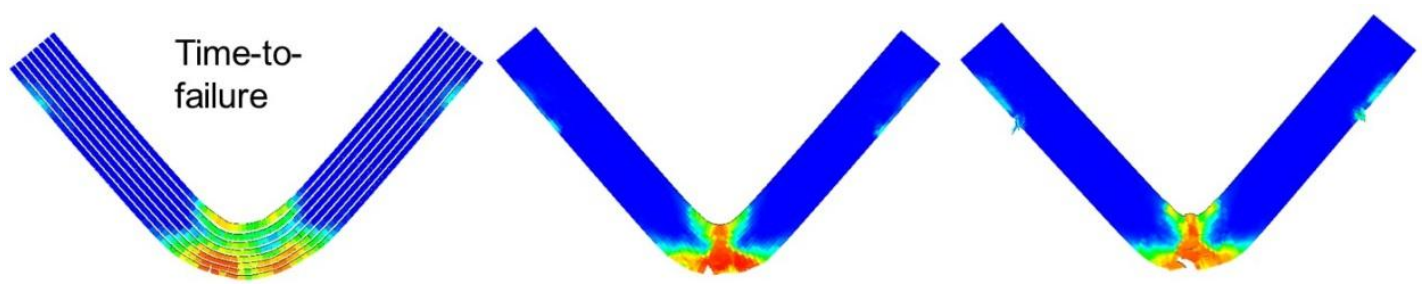

Figure 6: The same simulations as in Figure 3 except run using Kayenta's option for statistical variability and scale effects in strength. Mesh resolution increases from left to right.

To make the simulations complete faster, and also to investigate the rate effects on the specimen damage patterns, the initial research was conducted by running these simulations at a striker velocity 10 times that used in the experiments. Results using realistic striker speeds are discussed later in this section. Figure 7 shows the plot of damage using constant strain-to-failure of 0.3 . Moving from top to bottom in 
that figure corresponds to tripling the fracture speed, ${ }^{1}$ and moving from left to right corresponds to tripling the Weibull modulus (i.e., a decrease in variability). All of these simulations were run at the same mesh resolution. Figure 8 is the same as Figure 7, except the strain-to-failure was chosen to be 0.4. As seen in these figures, increasing FSPEED noticeably increases the size of the damage zone, whereas increasing the Weibull modulus (i.e., reducing variability) decreases the size of the damage zone.

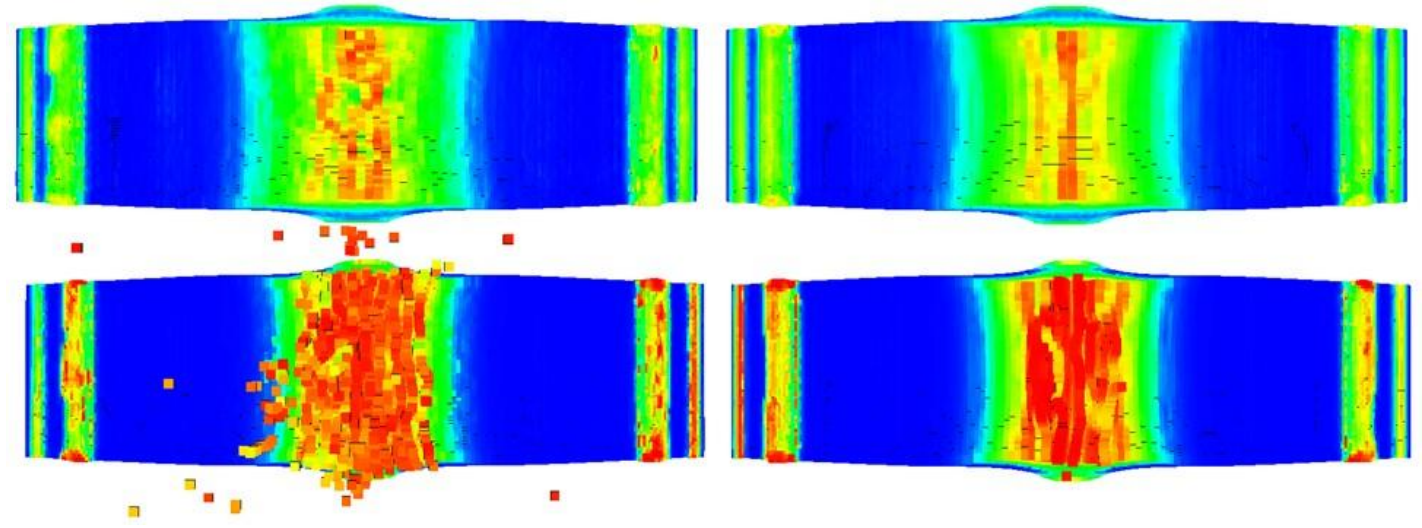

Figure 7: Plots of damage (red is damaged, and blue is intact material) using constant strain-to-failure softening model in Kayenta. Strain at failure is 0.3 . Moving from top to bottom corresponds to tripling the fracture speed (FSPEED parameter in Kayenta controls the rate of loss in strength in the material after softening starts to occur), and moving from left to right corresponds to tripling the Weibull modulus.

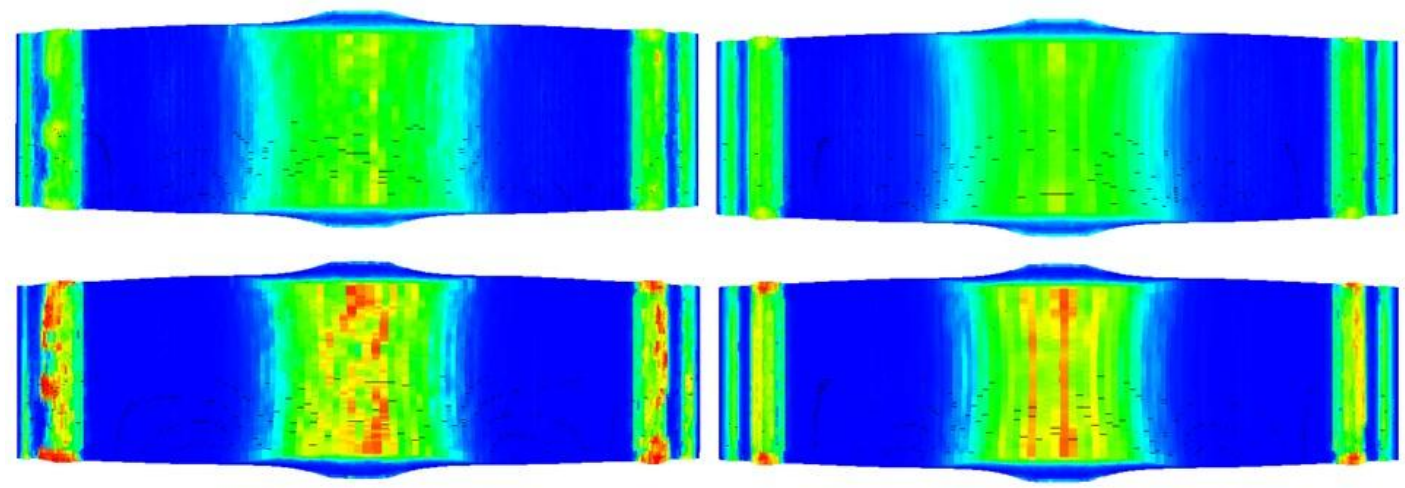

Figure 8: Plots of damage (red is damaged, and blue is intact material) using constant strain-to-failure softening model in Kayenta. Strain at failure is 0.4. Moving from top to bottom corresponds to tripling the fracture speed (FSPEED parameter in Kayenta controls the rate of loss in strength in the material after softening starts to occur), and moving from left to right corresponds to tripling the Weibull modulus.

A similar investigation was performed using Johnson-Cook damage parameters, using both Uintah's Johnson-Cook model and Kayenta with the Johnson-Cook option. In both cases, the Johnson-Cook damage model was predicting failure at total accumulated strain values close to 1.0. Since those values were actually not reached in the simulation, complete damage of the specimen was not predicted. Figure 9 shows the plot of scalar damage predicted by the Johnson-Cook fracture model. Moving from left to right corresponds to tripling the Weibull modulus. These parameters do not have any significant effect on the damage pattern.

\footnotetext{
${ }^{1}$ The Kayenta parameter called FSPEED controls the rate of loss in strength in the material after softening starts to occur. A large value of FSPEED makes Kayenta emulate the first Johnson-Holmquist (JH-1) model, while a lower value emulates the JH-2 model [39].
} 


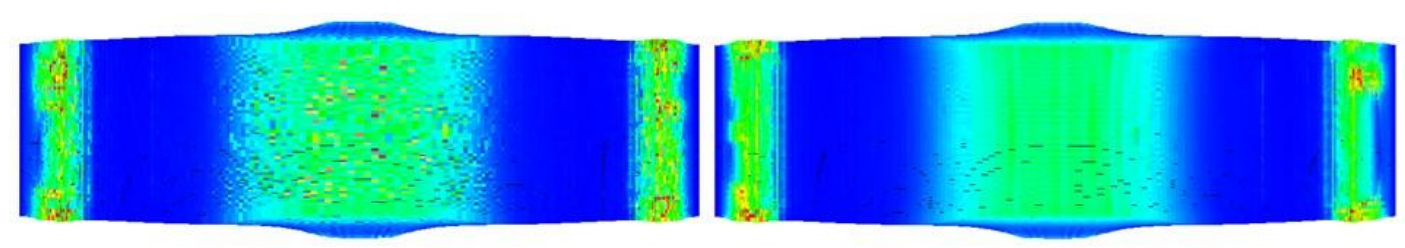

Figure 9: Plots of damage (red is damaged material, and blue is intact material) using Johnson-Cook softening model in Kayenta. Moving from left to right corresponds to tripling the Weibull modulus.

Figure 10 shows the damage patterns, the amount of energy absorbed, and the bend angle for the simulations that were run at 10 times the striker speed that was used in the experiments. The simulations were run using the constant-strain-failure softening model in Kayenta. Moving from left to right corresponds to tripling the Weibull modulus (i.e., a decrease in variability of strength), and moving from top to bottom corresponds to increasing failure strain from 0.3 to 0.4 . As seen in this figure, increasing the Weibull modulus or increasing failure strain decreases the size of the damage zone. The average bend angle calculated from these simulations was $7.7 \%$ higher than those observed in the experiments. The average amount of energy absorbed (Initial K.E. Striker - Final K.E. Striker) was $24 \%$ higher compared to the experiments. Keep in mind, however, that these simulations were run using a higher striking speed than were used in the actual experiments. Below, it is shown that realistic striker speeds give much better agreement with the data. Unsurprisingly, this shows that impact speeds have significant influence on absorbed energy and bend angle.
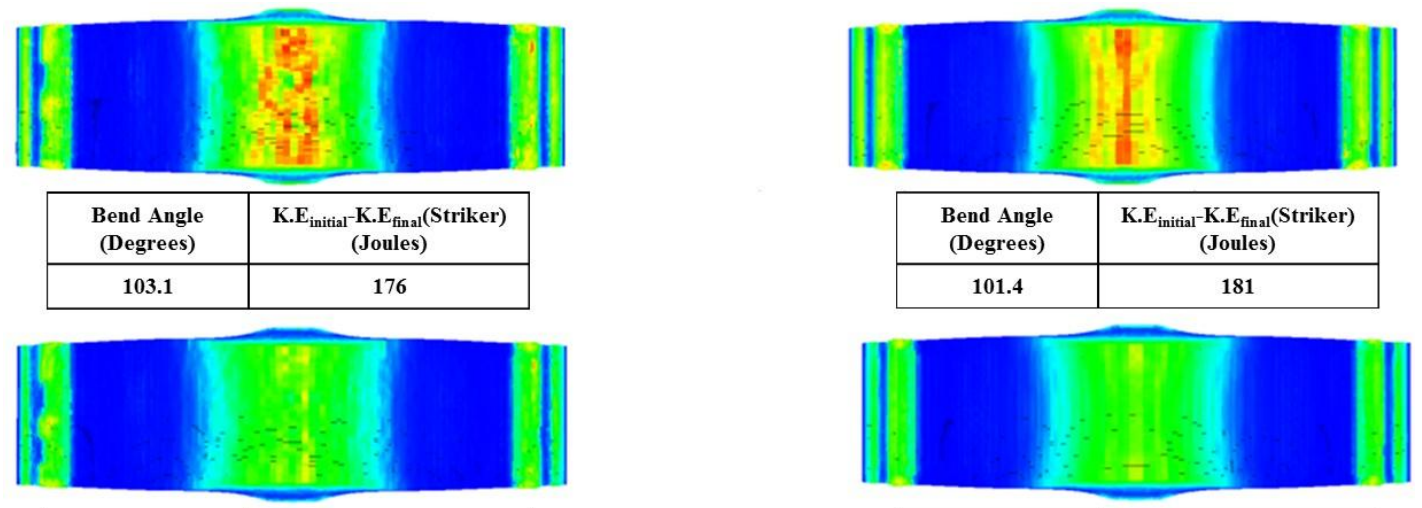

\begin{tabular}{|c|c|}
\hline $\begin{array}{c}\text { Bend Angle } \\
\text { (Degrees) }\end{array}$ & $\begin{array}{c}\text { K.E }_{\text {initial }} \text {-K.E }_{\text {final }} \text { (Striker) } \\
\text { (Joules) }\end{array}$ \\
\hline 101.7 & 181 \\
\hline
\end{tabular}

\begin{tabular}{|c|c|}
\hline $\begin{array}{c}\text { Bend Angle } \\
\text { (Degrees) }\end{array}$ & $\begin{array}{c}\text { K.E } \\
\text { initial } \\
\text { (Joules) }\end{array}$ \\
\hline 101.3 & 183 \\
\hline
\end{tabular}

Figure 10: Plots of damage (red is damaged material, and blue is intact material) using constant strain-tofailure softening with an artificially increased striker speed of $51.47 \mathrm{~m} / \mathrm{s}$. Moving from left to right corresponds to tripling the Weibull modulus (i.e., making the material more deterministic in its response), and moving from top to bottom corresponds to increasing failure strain from 0.3 to 0.4 .

Figure 11 shows the same simulations as in Figure 10, except the striker speed is the actual speed $(5.147 \mathrm{~m} / \mathrm{s})$ calculated in the experiments. The trend in damage patterns is similar to Figure 10, but quantitative results were much closer to experimental values. The average bend angle calculated from these simulations was $0.4 \%$ higher than those observed in the experiments. The average amount of energy (Initial K.E. Striker - Final K.E. Striker) absorbed was 1.2\% lower compared to the experiments. 

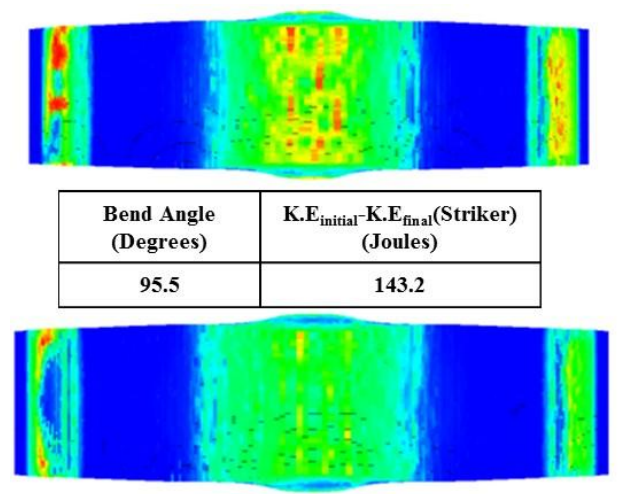

\begin{tabular}{|c|c|}
\hline $\begin{array}{c}\text { Bend Angle } \\
\text { (Degrees) }\end{array}$ & $\begin{array}{c}\text { K.E }_{\text {initial }}-\text { K.E }_{\text {final }} \text { (Striker) } \\
\text { (Joules) }\end{array}$ \\
\hline 95.3 & 144.2 \\
\hline
\end{tabular}
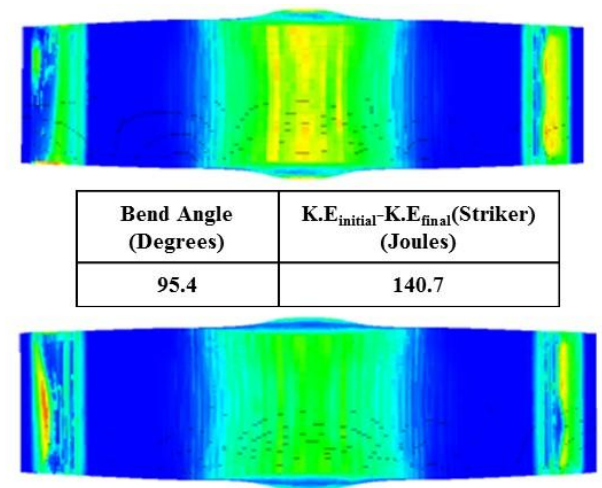

\begin{tabular}{|c|c|}
\hline $\begin{array}{c}\text { Bend Angle } \\
\text { (Degrees) }\end{array}$ & $\begin{array}{c}\text { K.E }_{\text {initial }} \text {-K.E }_{\text {final }} \text { (Striker) } \\
\text { (Joules) }\end{array}$ \\
\hline 95.6 & 144.4 \\
\hline
\end{tabular}

Figure 11: Plots of damage (red is damaged material, and blue is intact material) using constant strain-tofailure softening model with striker speed of $5.147 \mathrm{~m} / \mathrm{s}$. Moving from left to right corresponds to tripling the Weibull modulus, and moving from top to bottom corresponds to increasing failure strain from 0.3 to 0.4 .

All of the simulations presented so far were run at one mesh size. When refining the mesh, scale effects are expected to be needed for convergence with mesh refinement. In particular, smaller domains (e.g., smaller elements) are stronger on average and also more variable so that, in principle, the probability of failure initiation of a given domain is insensitive to whether that domain is subdivided into few or many elements [21]. Scale effects for progression of failure following initiation remain a subject of active research [22]. In light of a known tendency for damage simulations to be mesh sensitive [23], [24], two convergence studies were performed on the unnotched Charpy problem using Kayenta's strainto-failure option (with the failure strain chosen to be 0.3). The speed of the striker was the same as in the experiments. In the first convergence study, strength was statistically variable, but no scale effects were imposed. The three cell spacings and the absorbed energy are listed in the Table 1. The second convergence study includes scale effects in order to assess whether or not they tend to at least mitigate mesh sensitivity.

Table 1: Mesh configurations and the amount of energy absorbed using statistical variability in strength with no scale effects.

\begin{tabular}{|l|l|l|l|l|}
\hline Mesh & $\begin{array}{l}\text { Cell spacing } \\
{[x, y, z](\mathrm{cm})}\end{array}$ & $\begin{array}{l}\text { Initial K.E. } \\
\text { (Joules) }\end{array}$ & $\begin{array}{l}\text { Final K.E. } \\
\text { (Joules) }\end{array}$ & $\begin{array}{l}\text { ASTM Energy absorbed by } \\
\text { the specimen (Joules) }\end{array}$ \\
\hline \hline Mesh 1 & $\begin{array}{l}0.125,0.125, \\
0.125\end{array}$ & 358 & 192 & 166 \\
\hline Mesh 2 & $\begin{array}{l}0.0625,0.0625, \\
0.0625\end{array}$ & 365 & 208 & 157 \\
\hline Mesh 3 & $\begin{array}{l}0.03125,0.0625, \\
0.0625\end{array}$ & 365 & 238 & 127 \\
\hline
\end{tabular}

The plot for the time history of the K.E. of the striker in the convergence study without scale effects can be seen in Figure 12. The rate of K.E. absorbed by the specimen is almost constant for mesh 1 and mesh 2. For mesh 3, the rate of absorbed K.E. decreases during the simulation and tends towards a steady-state value, thus indicating that the fine mesh is predicting less striker K.E. being absorbed by the specimen. The results show mesh convergence prior to specimen damage, but nonconvergence during the softening phase. Such behavior is a typical and as-yet unresolved problem for softening models that lack scale effects, thus motivating us to perform the convergence study again using both statistical variability in strength and scale effects to see if this issue could be resolved. The resulting time histories of the K.E. of the striker can be seen in Figure 13. 


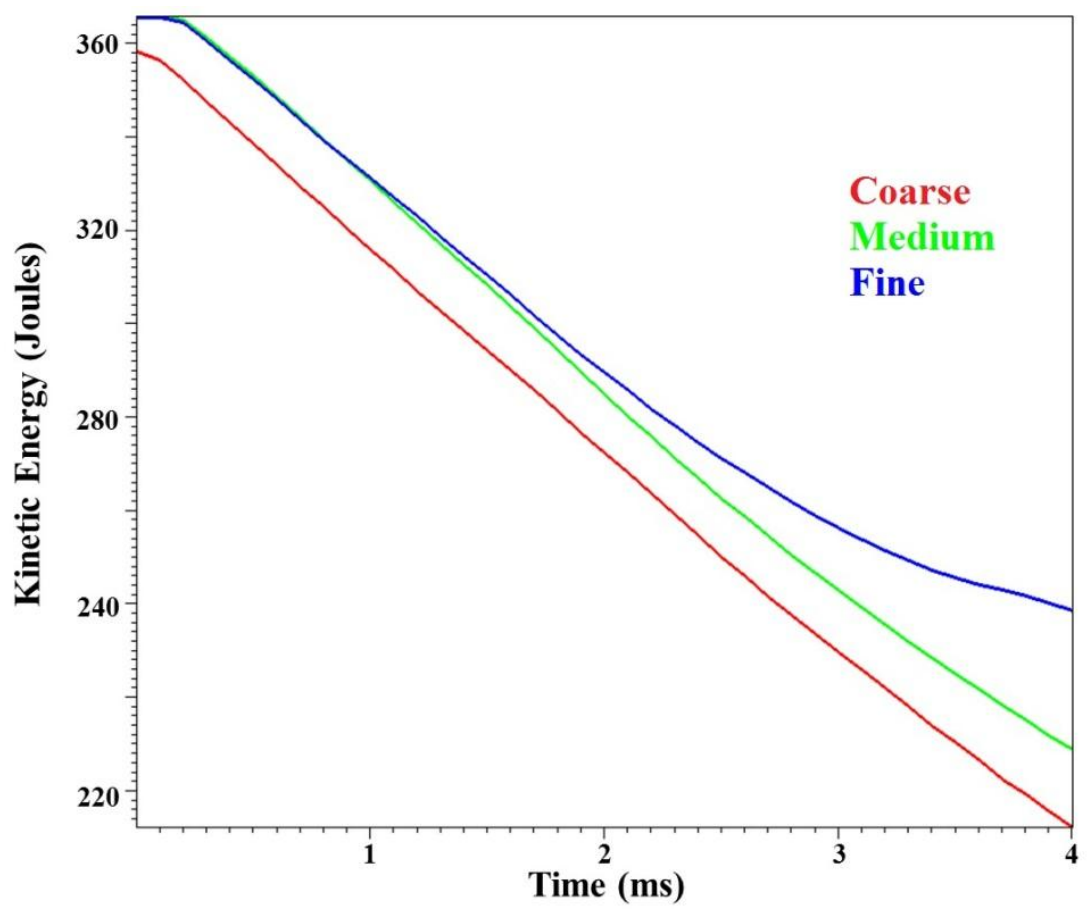

Figure 12: Time history of the K.E. of the striker for three meshes using statistical variability in strength with no scale effects.

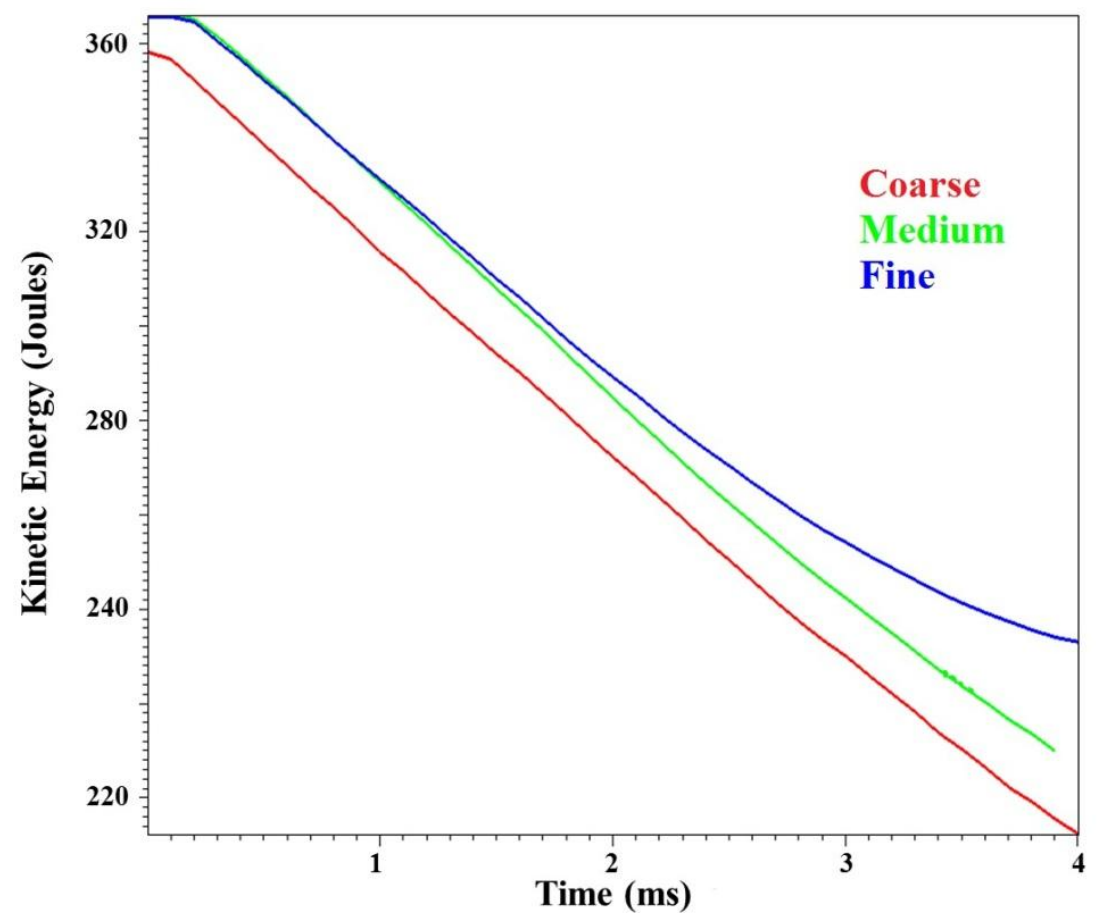

Figure 13: Time history of the K.E. of the striker for three meshes using statistical variability in strength and scale effects.

Both cases show similar trends, with inclusion of scale effects only slightly improving convergence during the later times. To illustrate the influence of scale effects, Figure 14 shows the color plots of damage for both cases. Mesh resolution increases from top to bottom. The left column corresponds to the simulations using statistical variability in strength without scale effects, and the right column corresponds to the simulations using both statistical variability in strength and scale effects. For lower and medium 
mesh resolutions, the influence of scale effects is negligible, but the influence of scale effects can be clearly seen in the highly refined case. The damage appears to be concentrating at the bottom-center of the specimen using scale effects. It is evident that further mesh refinement is warranted to check if this trend persists.
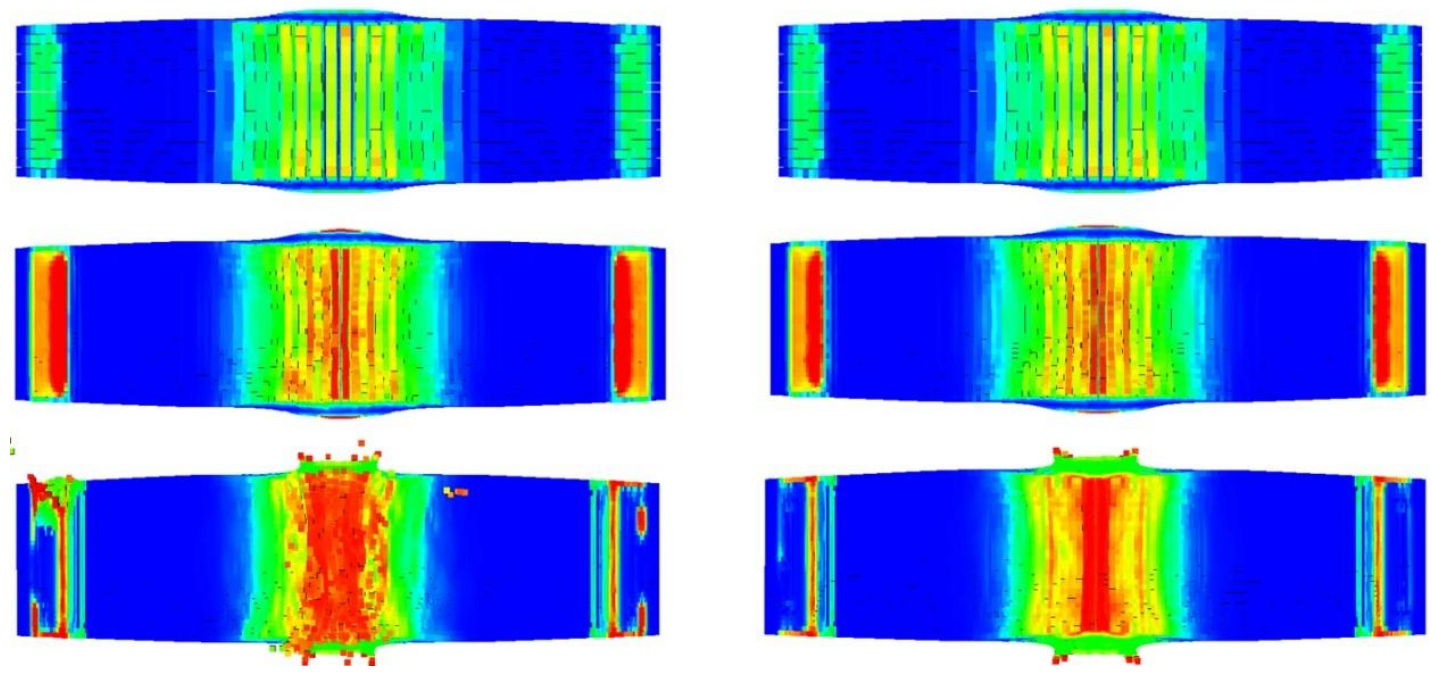

Figure 14: Color plots of damage. Blue is intact material and red is completely damaged material. Mesh resolution increases from top to bottom. The left column corresponds to the simulations using statistical variability in strength without scale effects and the right column corresponds to the simulations using both statistical variability in strength and scale effects.

\section{Experimental data on Taylor-anvil impact test}

Taylor impact specimens from tests conducted at the INL National Security Test Range were employed to compare the experimental and computed deformation and fracture of a titanium alloy. These Taylor cylinder specimens were impacted while developing an optimization-based method to identify (rather than confirm) material constitutive parameters from sparse Taylor data [25]. INL's Taylor apparatus comprises a large-bore, powder-driven gun firing saboted sub-caliber test specimens into a polished AR-400 steel anvil. Specimen impact velocities are recorded with a two-stage light-screen chronograph, while impacts are filmed on 2 axes with high-speed phantom cameras. This setup allows larger specimens to be tested at higher impact speeds than may be typical-important for materials where size effects are important, and for very hard materials. An annotated photograph of the apparatus is shown in Figure 15.

The specimens are Ti-6Al-4V material machined from rolled plate stock 1"thick. To see whether rolling texture affects the deformation and fracture, specimens were cut both along and across the rolling direction. All specimens were cut in the plane of the plate. The rolling direction was tracked throughout the fabrication process and marked with a red arrow on each test specimen. Of the two specimens of interest, item $42(321 \mathrm{~m} / \mathrm{s})$ was aligned with the rolling direction, and item $58(393 \mathrm{~m} / \mathrm{s})$ was transverse. 


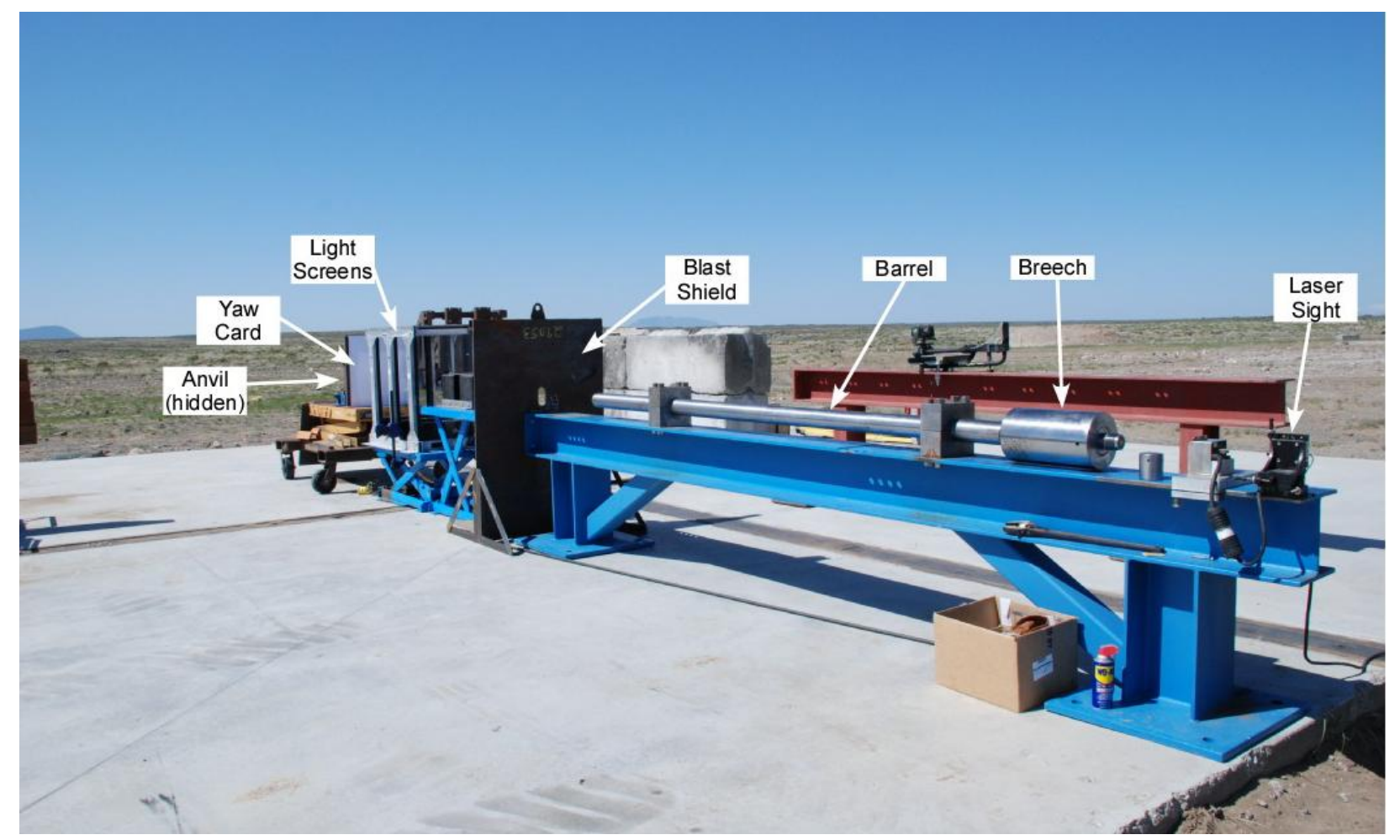

Figure 15: INL Large-bore Taylor Apparatus [25].

Two specimens were selected for study which had been impacted at speeds of $321 \mathrm{~m} / \mathrm{s}$ and $393 \mathrm{~m} / \mathrm{s}$. The material was Ti6Al4V and the specimen dimensions were $4.21 \mathrm{~cm}$ long and $1.27 \mathrm{~cm}$ in diameter. The average mass of the specimens was $24.21 \mathrm{gm}$. Figures 16-17 show the damage patterns observed in the experiments. At $321 \mathrm{~m} / \mathrm{s}$, a couple of cracks at approximately $45^{\circ}$ and two small fragments were separated from the bottom of the specimen. At $393 \mathrm{~m} / \mathrm{s}$, all fragments were completely separated from the bulk of the specimen.

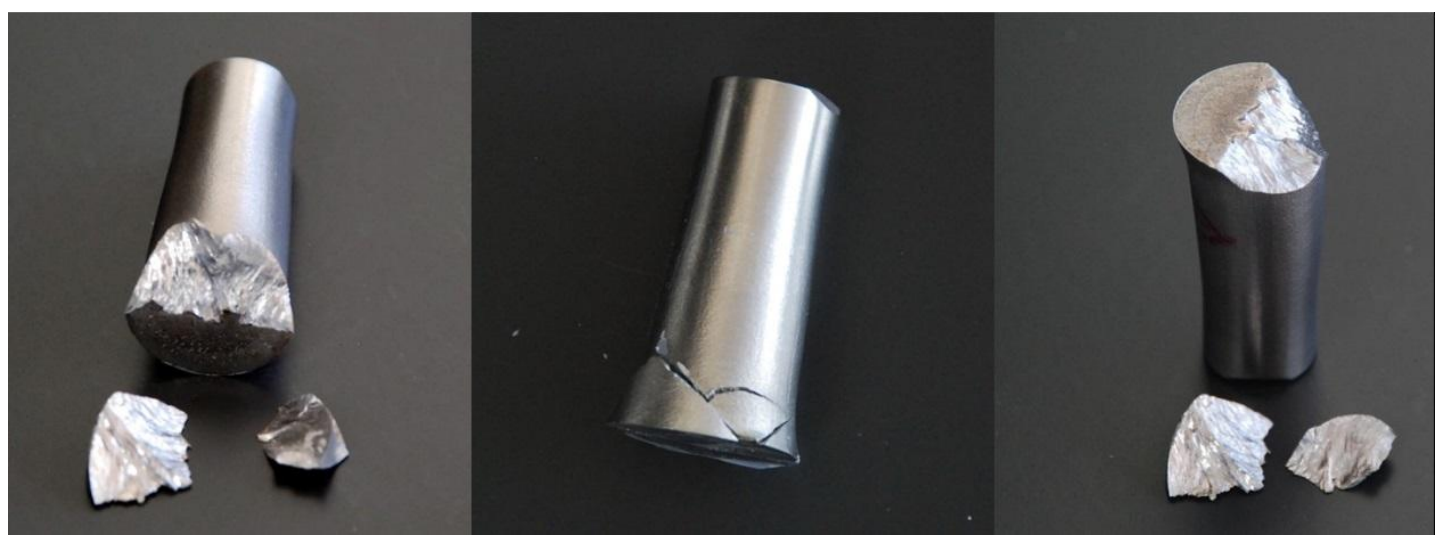

Figure 16: Experimental observations of damage patterns at specimen speed of $321 \mathrm{~m} / \mathrm{s}$. 


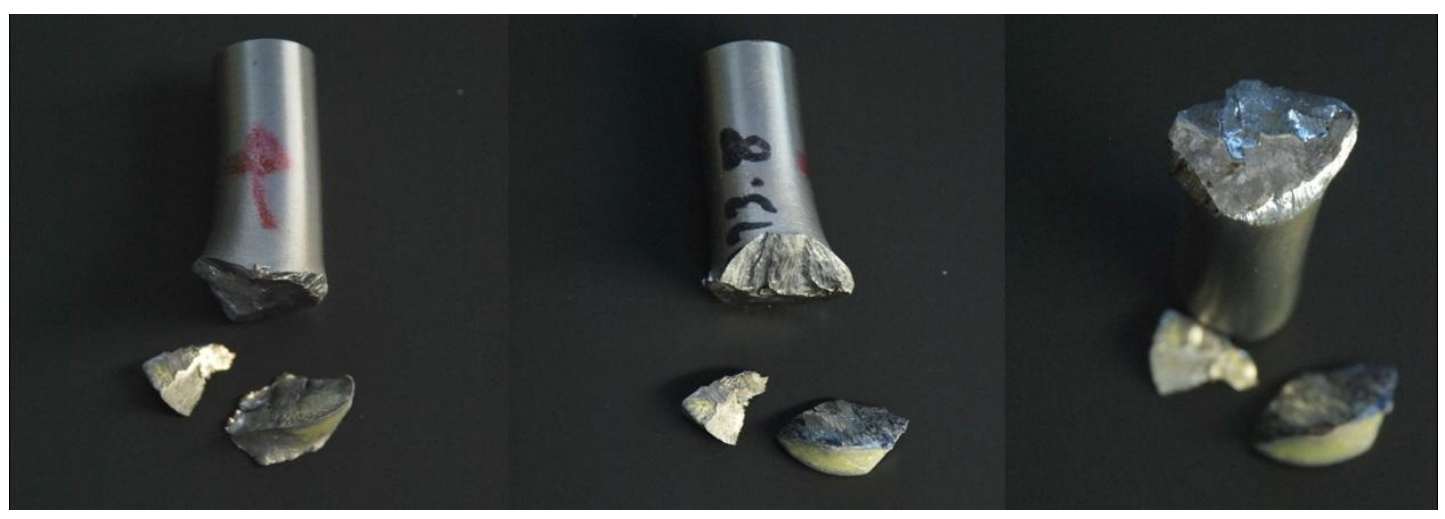

Figure 17: Experimental observations of damage patterns at specimen speed of $393 \mathrm{~m} / \mathrm{s}$.

\section{Numerical simulations of Taylor-anvil impact}

As this test involves large plastic deformations and damage at high rates, the constitutive model should be rate-dependent. Kayenta includes two forms of rate dependence. For softening, a scaledependent time-to-failure option provides user control for the rate at which damage propagates. The plasticity component of Kayenta further provides a conventional Duvaut-Lions viscoplastic formulation [26] to account for rate effects at high-rate loading. Since Kayenta's Duvaut-Lions rate dependence option had not been previously tested or used in combination with softening, a verification of the implementation of rate-dependence was performed before using both features for validation.

\subsection{Verification of rate dependence, hardening and softening in Kayenta}

A uniaxial-strain single-element test was run at low and high strain rates for von-Mises plasticity to verify the effect of strain rates on the results. The following cases were used for the trend tests:

- Strain-to-failure softening with no rate dependence

- No softening with rate dependence

- Strain-to-failure softening with rate dependence

- Strain-to-failure softening with rate dependence and hardening

For the verification tests described below, the pertinent parts of the input deck are shown in Table 2 .

Table 2: Input data used for single-element verification tests

\begin{tabular}{|l|l|l|l|}
\hline Yield stress in shear & Rate dependence & $\begin{array}{l}\text { Softening } \\
\text { (Johnson- Cook Failure } \\
\text { model) }\end{array}$ & Hardening \\
\hline$A_{1}=570 \mathrm{MPa}$ & $T_{1}=1 \times 10^{-6} \mathrm{~S}, T_{2}=0$ & $D_{1}=-0.81$, & $\mathrm{HC}=1 \mathrm{GPa}$, \\
& & $\mathrm{D}=1.18$, \\
& & $\mathrm{RN}=-0.25 \mathrm{GPa}$ \\
& & \\
& & $D_{4}=-0.015$, \\
& $D_{5}=2.10$ & \\
\hline
\end{tabular}




\subsubsection{Strain-to-failure softening without rate dependence}

This section discusses the uniaxial-strain single-element test for von-Mises plasticity with strain-tofailure softening and no rate dependence. The element was stretched until it failed. From Figure 19, it can be seen that the slope discontinuity happens at the right location when it yields ${ }^{2}$, and as COHER (which is the ratio of the current strength to the initial strength) goes from 1 to zero, shown in Figure 18, the element loses strength accordingly. A similarly appropriate trend can be seen for the same problem at higher strain rates in Figures 20 and 21. The plots at the two different strain rates are identical because this problem is with strain-to-failure softening only, and not rate dependence.

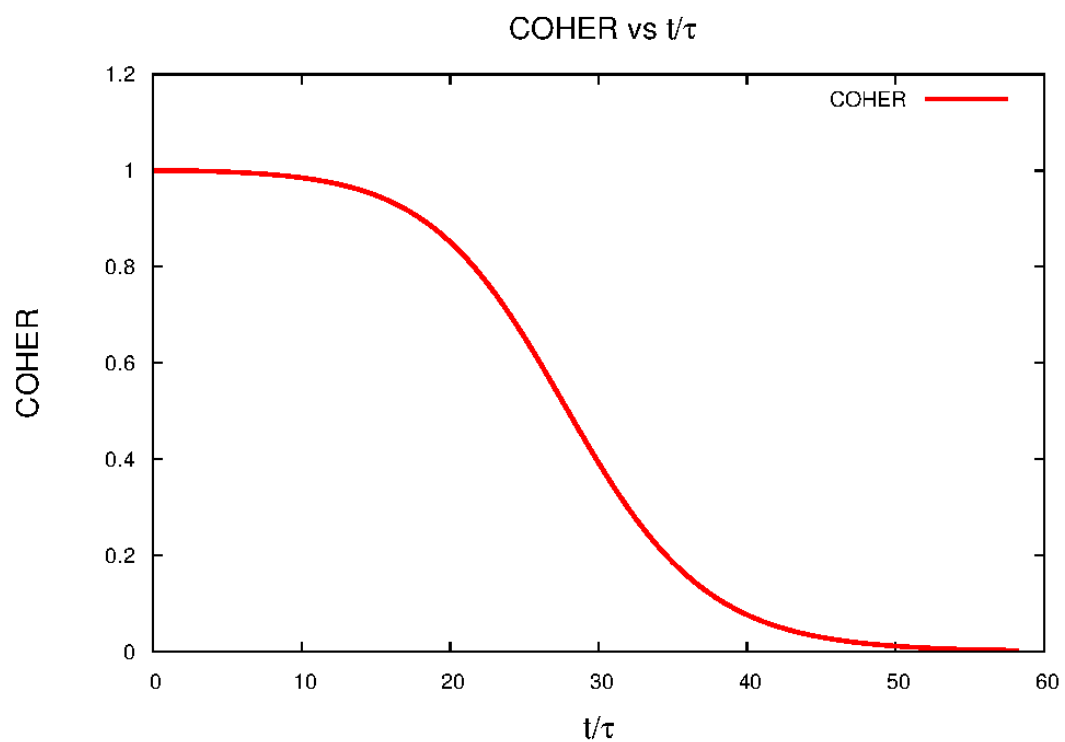

Figure 18: Normalized time history of the parameter COHER at low strain rates (softening only).

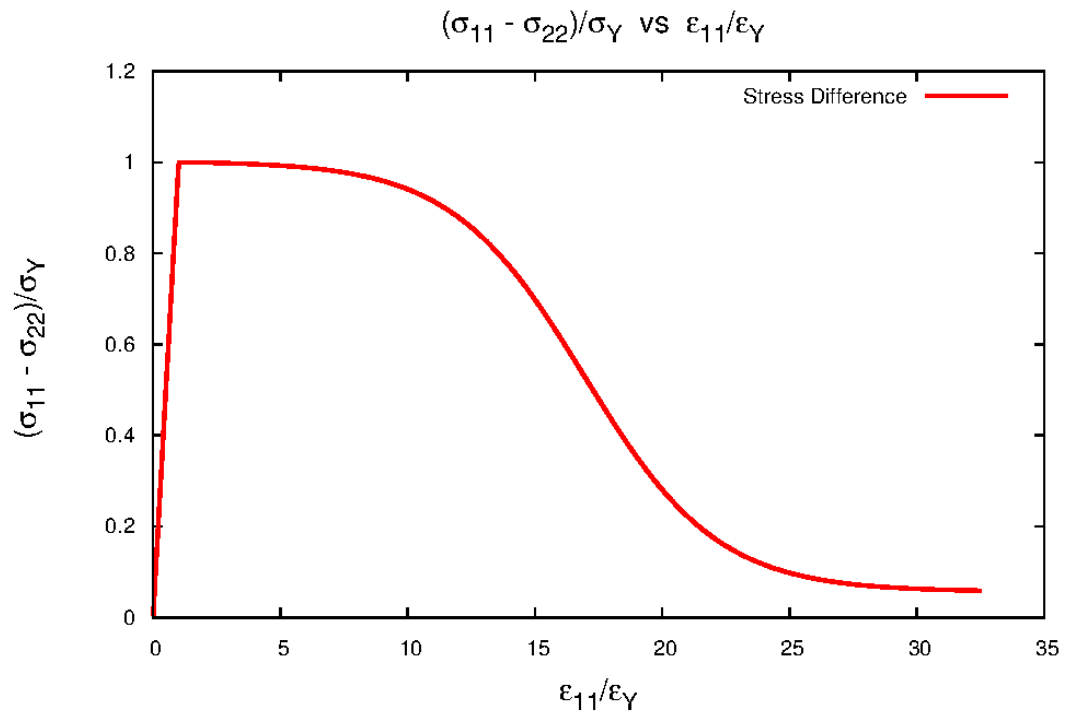

Figure 19: Normalized stress difference vs. normalized strain at low strain rates (softening only).

\footnotetext{
${ }^{2}$ That is, noting that the axes are nondimensionalized by the values of stress and strain at yield, the graph should exhibit a slope discontinuity exactly at the point $(1,1)$
} 


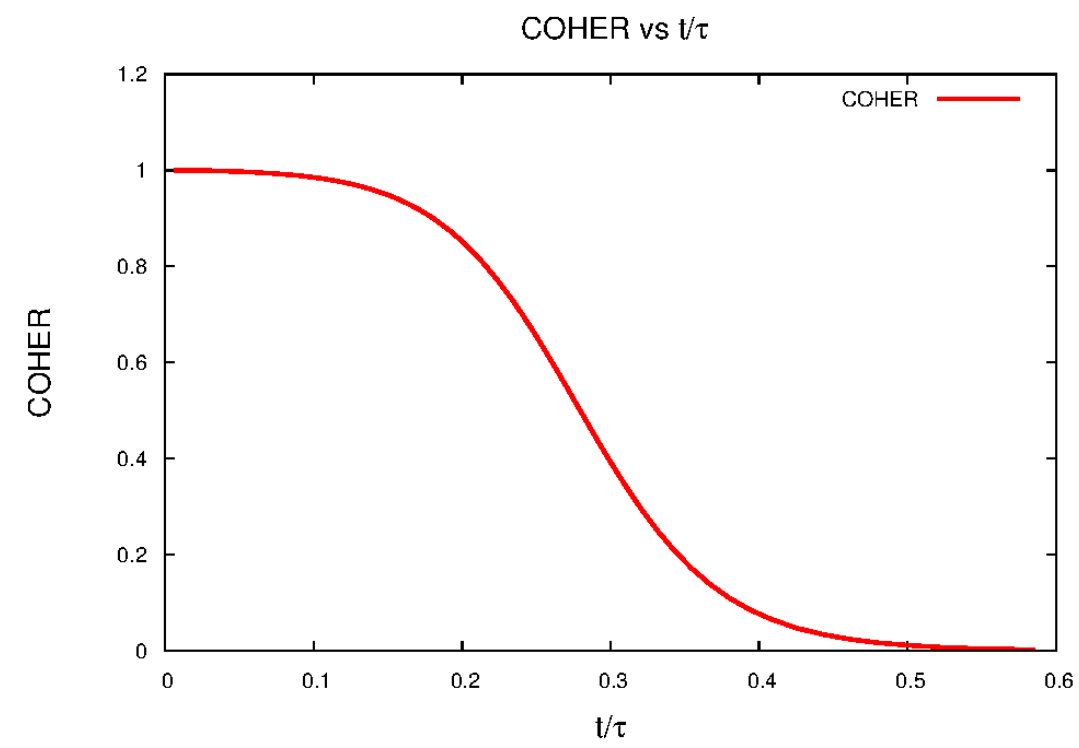

Figure 20: Normalized time history of the parameter COHER at high strain rates (softening only).

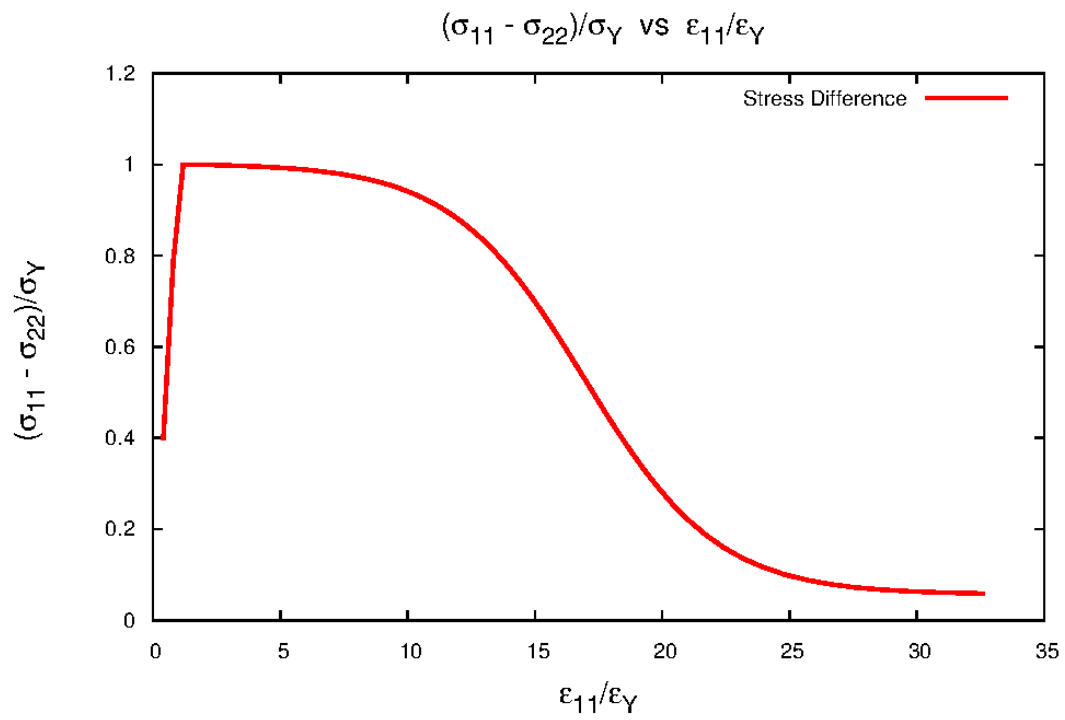

Figure 21: Normalized stress difference vs. normalized strain at high strain rates (softening only).

\subsubsection{No softening with rate dependence}

This section discusses the same test but with rate dependence and no softening. Figures 22 and 23 verify that, under very small strain rates, the effect of rate dependence is negligible. Since there is no softening, the COHER will stay at unity, and the normalized stress difference stays at yield. When run at higher strain rates, Figure 24 shows no change in COHER, which is correct as there is no softening, and Figure 25 shows the apparent increase in yield. ${ }^{3}$

\footnotetext{
${ }^{3}$ This is seen by the fact that the ordinate values exceed unity. Also, the post-yield decrease in stress is an artifact of running the simulations at a constant stretch rate, which results in a nonconstant strain rate that is higher at the beginning of loading than at the end (so the model is showing an appropriate decrease in apparent strength).and less damage compared to one without rate dependence. Additionally, when hardening is activated, the specimen should (and does) predict even less damage as evident in Figure 32. These are trend tests only; a more realistic simulation must naturally include statistics and scale effects of failure properties.
} 


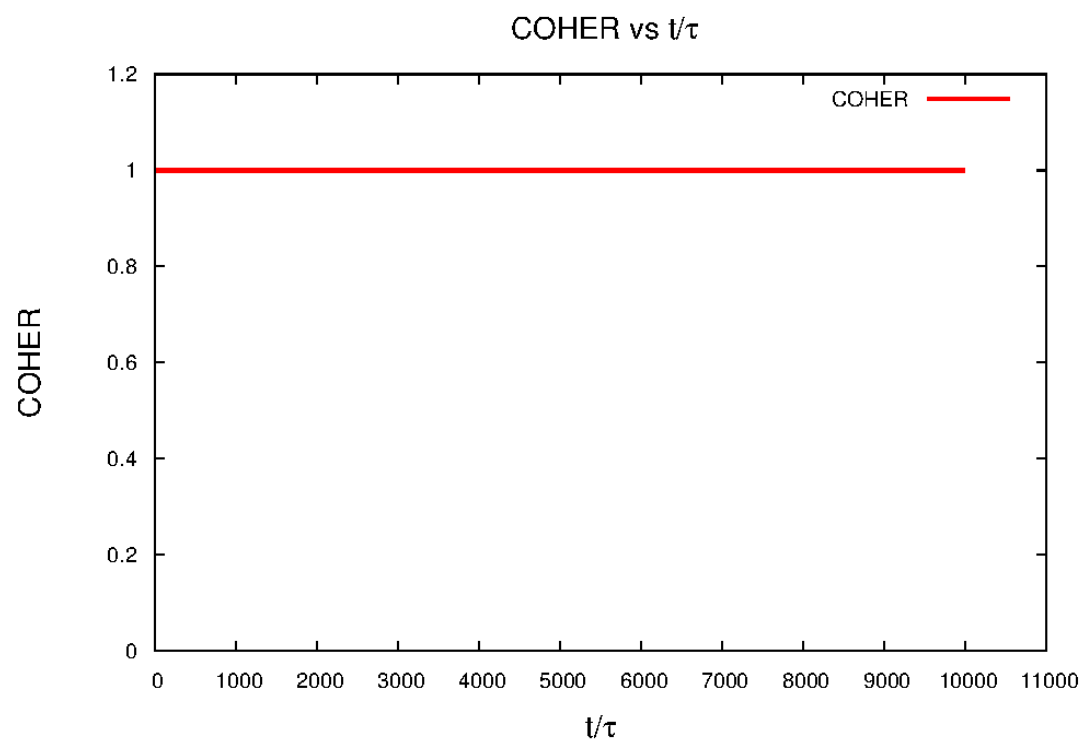

Figure 22: Normalized time history of the parameter COHER at low strain rates (rate dependence only).

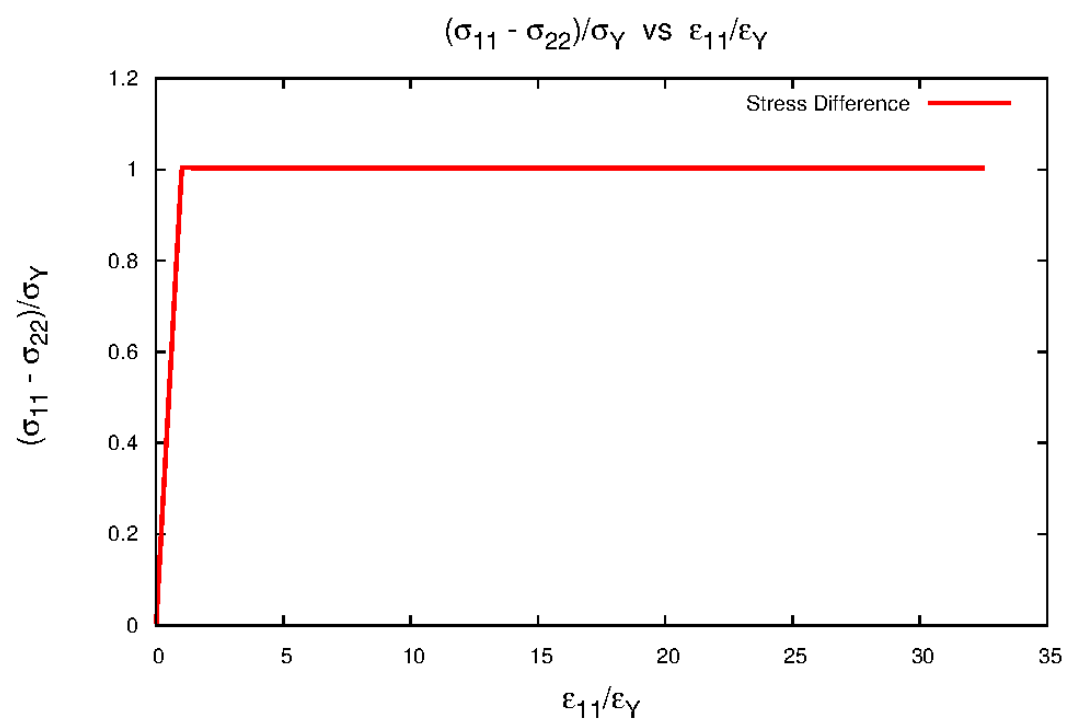

Figure 23: Normalized stress difference vs. normalized strain at low strain rates (rate dependence only). 


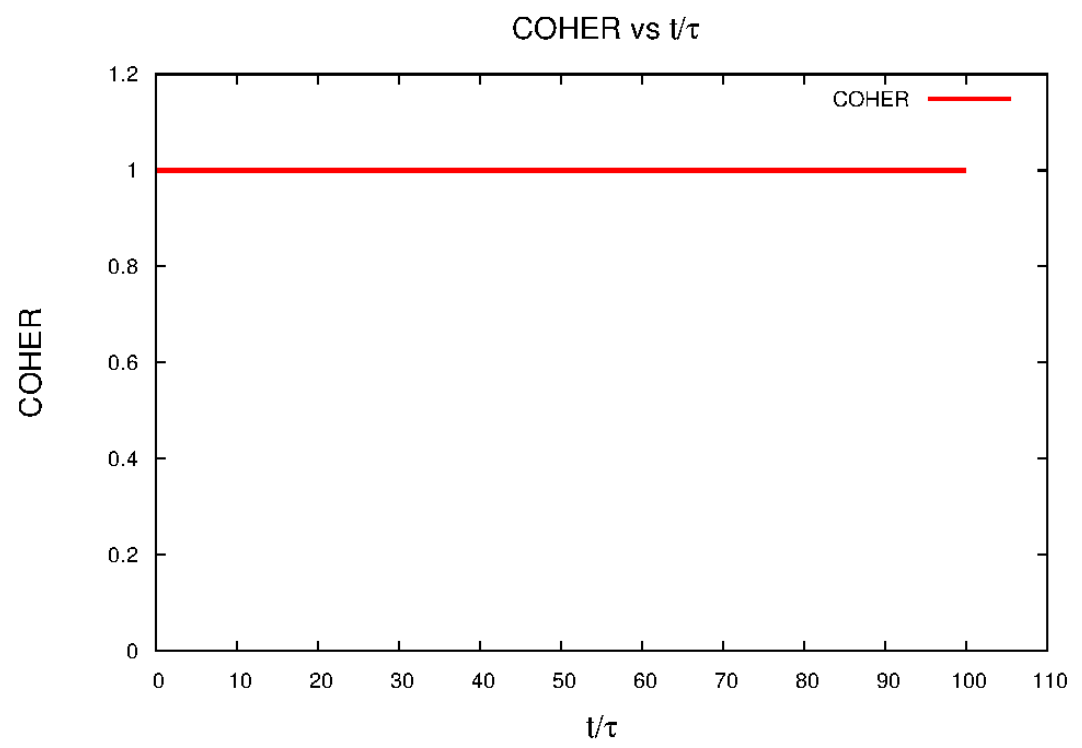

Figure 24: Normalized time history of the parameter COHER at high strain rates (Rate dependence only).

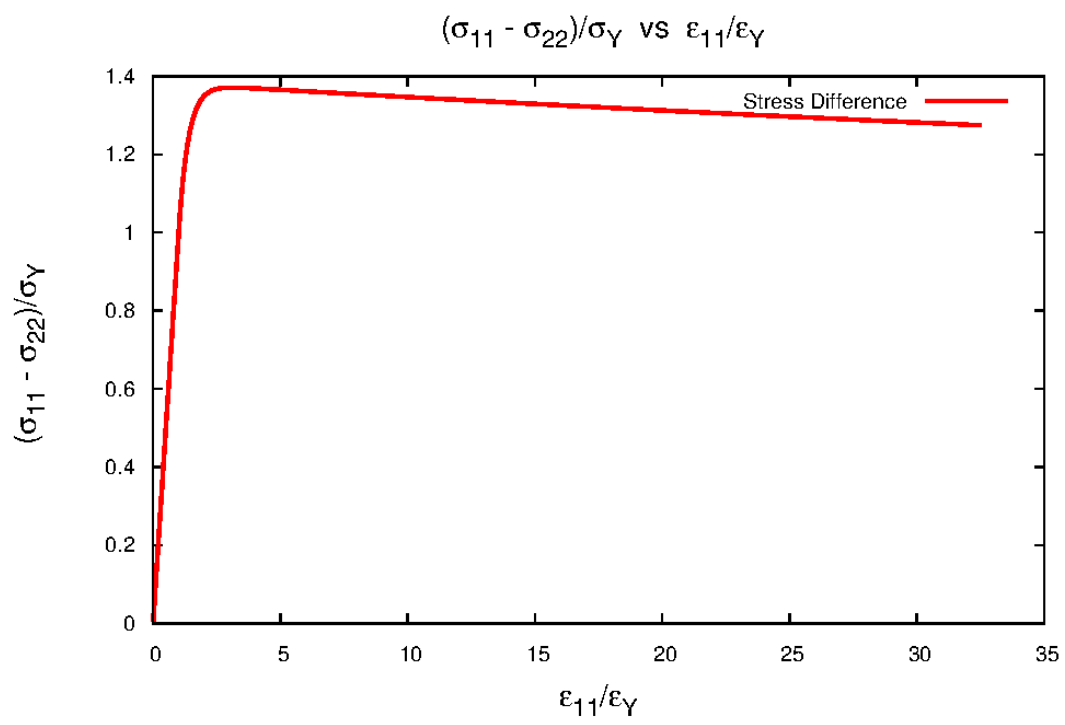

Figure 25: Normalized stress difference vs. normalized strain at high strain rates (rate dependence only). 


\subsubsection{Strain-to-failure softening with rate dependence}

This section discusses the results of the single-element test with both rate dependence and strain-to-failure softening. At low strain rates where rate dependence is negligible, Figure 27 is similar to Figure 19 where both lose strength in unison as COHER drops according to Figure 26.

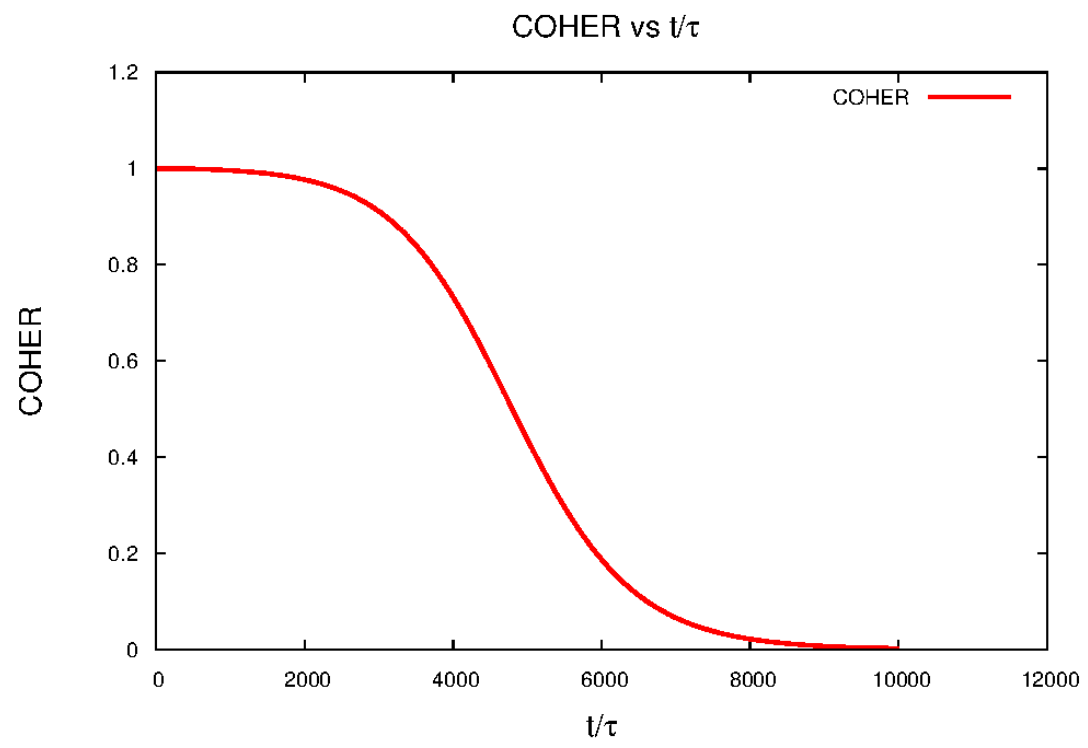

Figure 26: Normalized time history of the COHER at low strain rates (both rate dependence and strain-tofailure softening).

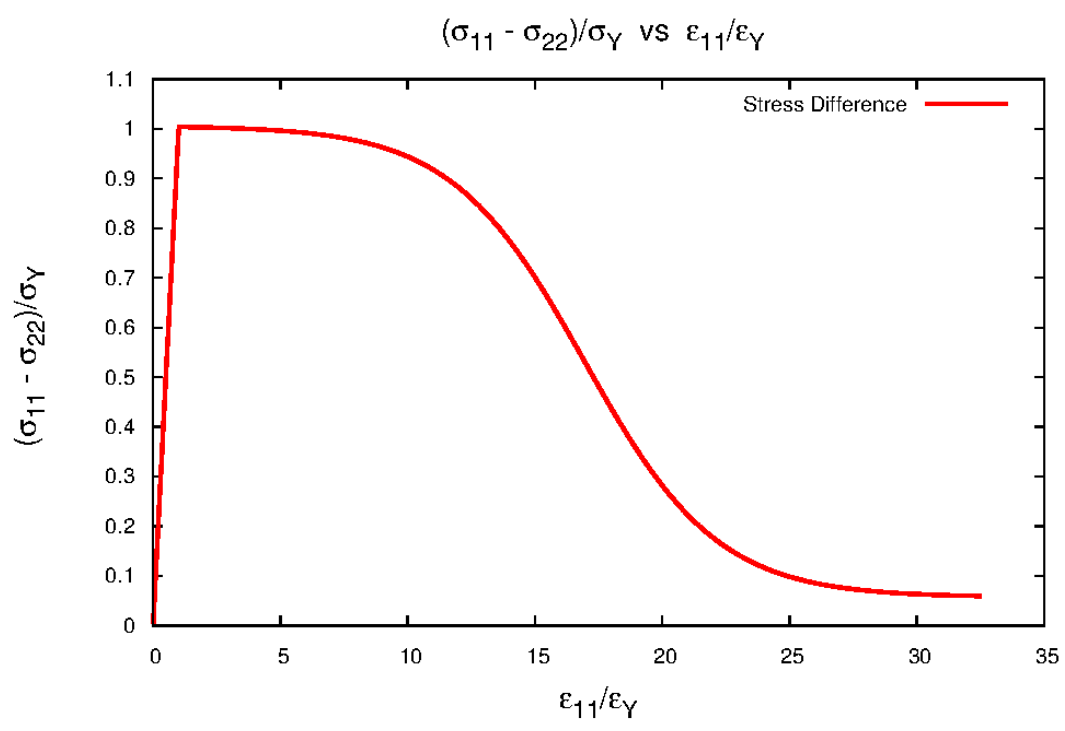

Figure 27: Normalized stress difference vs. normalized strain at low strain rates (both rate dependence and strain-to-failure softening).

At higher strain rates and with large enough deformation for the element to get damaged, both softening and rate dependence play a significant role. Figure 28 shows the normalized time history of COHER at higher strain rates, where the element is completely damaged (COHER approaches zero). Figure 29 shows that the normalized stress difference yields above the value of 1 , which indicates a higher apparent yield strength than the quasistatic yield strength. Also, the element loses strength according to COHER. These trends tests were performed to build confidence in the implementation of rate dependence with softening in Kayenta. 


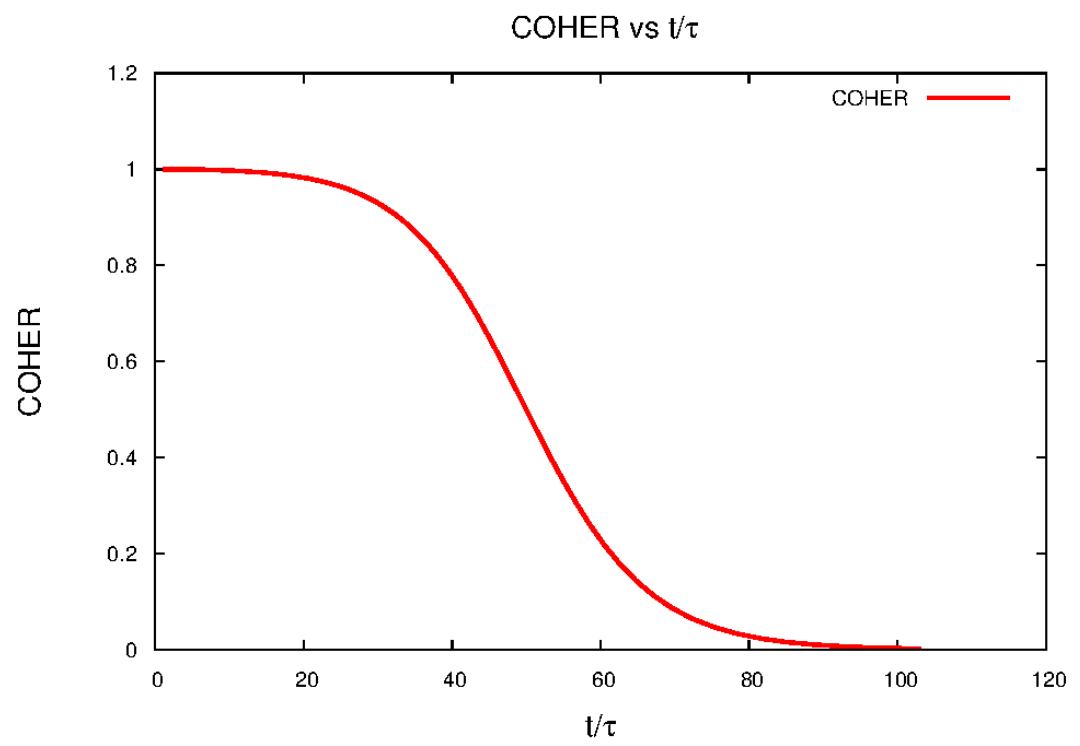

Figure 28: Normalized time history of the parameter COHER at high strain rates (both rate dependence and strain-to-failure softening).

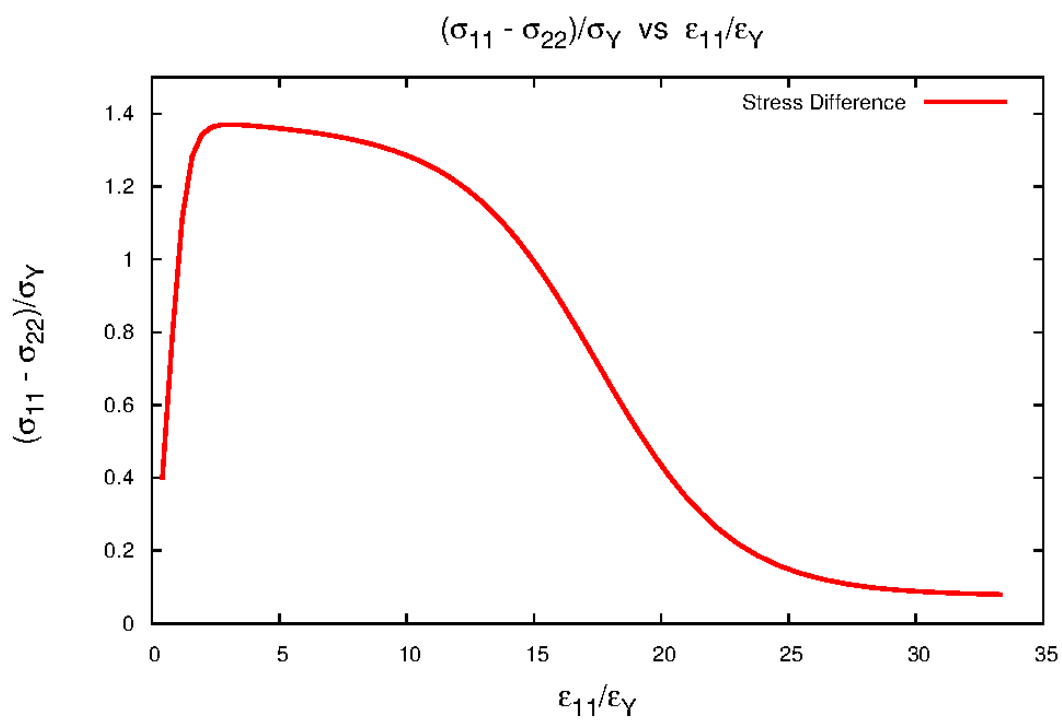

Figure 29: Normalized stress difference vs. normalized strain at high strain rates (both rate dependence and strain-to-failure softening).

\subsubsection{Strain-to-failure softening with rate dependence and hardening}

Figures 30-31 show the single-element test results using hardening, rate dependence, and softening. The figures show COHER (ratio of damaged strength to initial strength) vs. normalized time, and normalized stress difference vs. normalized strain using hardening, rate dependence, and softening, respectively. The stress response exhibits rate dependence (i.e., has an apparent normalized yield stress greater than 1), hardening (nonzero slope post yield), and softening (reduction in strength and stiffness). Also, as COHER approaches zero, the stress-difference approaches the failed strength, as desired. 


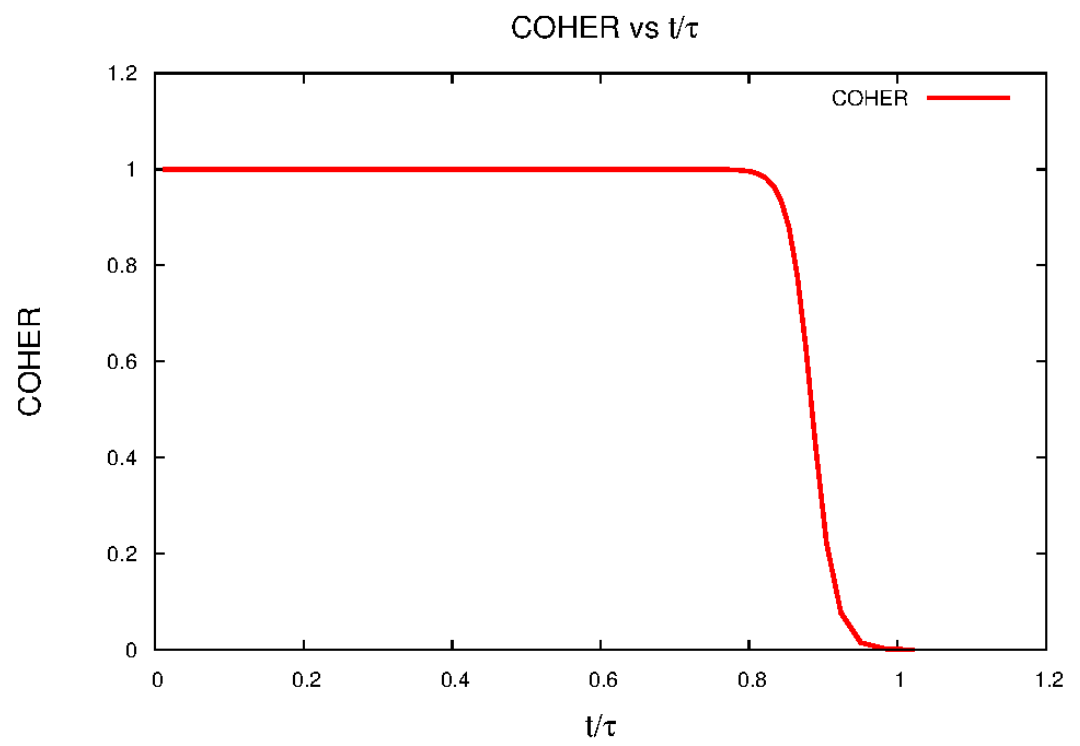

Figure 30: Normalized time history of the COHER at high strain rates (rate dependence, hardening and softening).

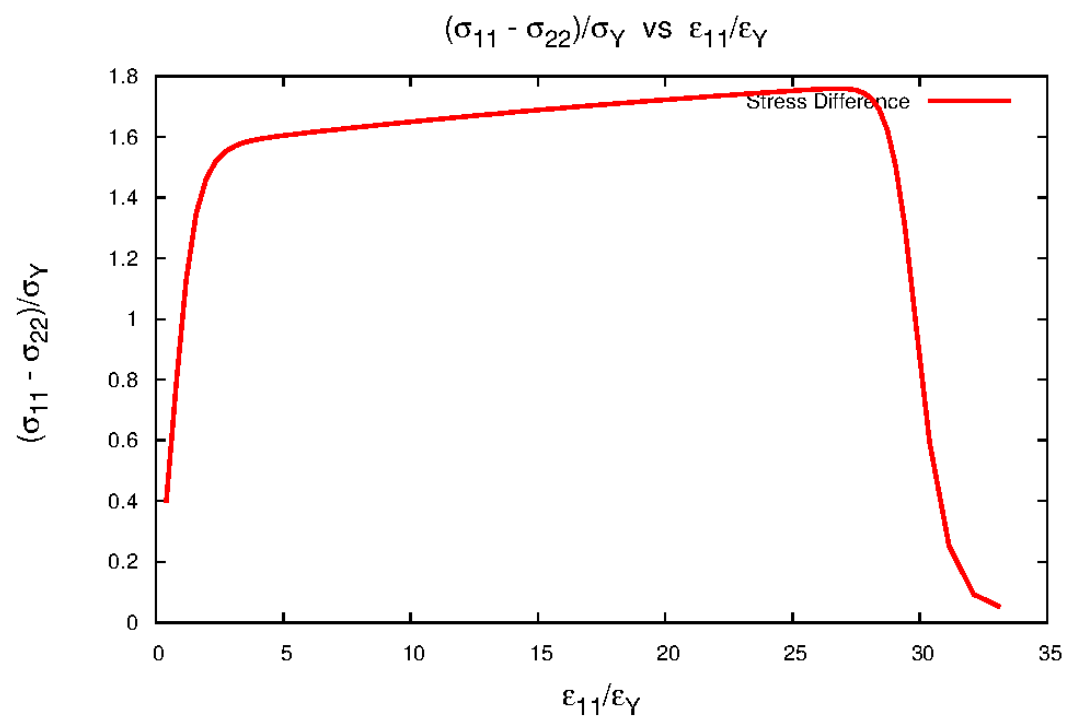

Figure 31: Normalized stress difference vs. normalized strain at high strain rates (rate dependence, hardening and softening).

After the constitutive model was verified using the single-element tests, Taylor-anvil impact simulations were run switching on each of the features i.e., softening, rate dependence, and hardening. Figure 32 shows the simulation results when each feature was activated. Moving from left to right shows qualitatively expected trends. In particular, at high rates, when rate dependence is activated, the specimen should predict less yielding. 

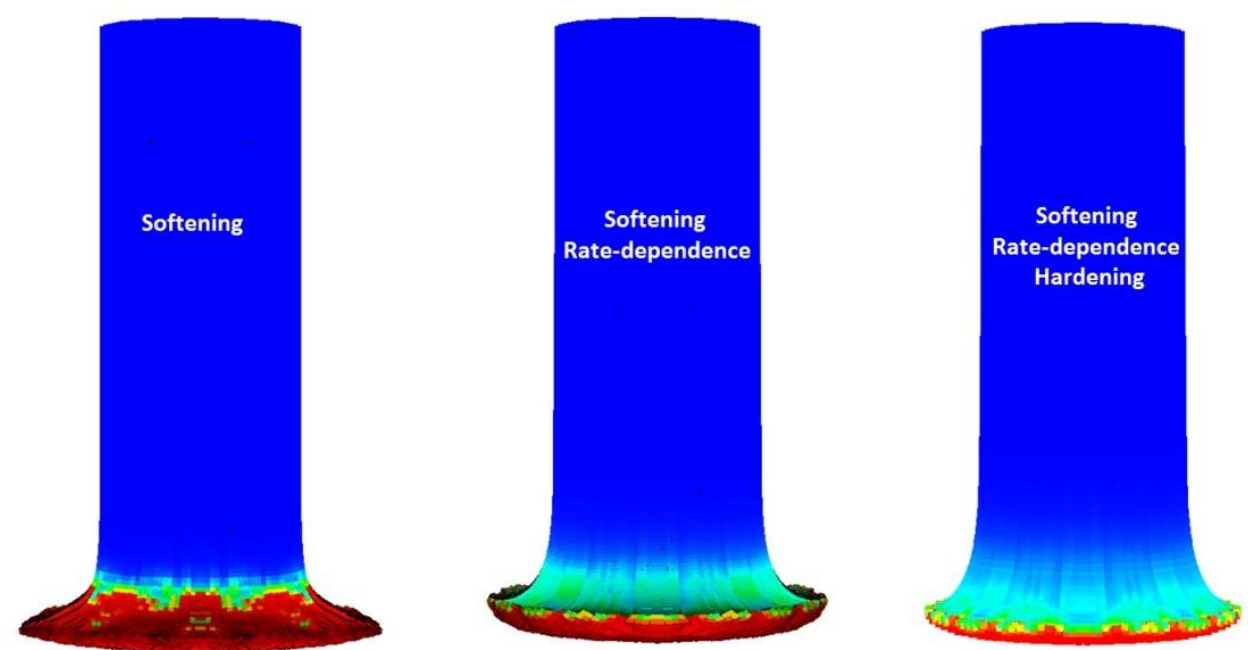

Figure 32: Trend tests on Taylor-anvil impact simulations using deterministic material properties (i.e., no statistical variability or scale effects). Blue is intact material and red is damaged material.

\subsection{Calibrating rate-dependence parameters for Kayenta}

Experimental data for rate-dependence of strength of Ti6Al4V is abundantly available in the literature [27], [28], [29], [30]. For metals, the yield and flow stresses are shown to be sensitive to hydrostatic pressures [31], [32]. For Ti6Al4V, up to confining pressures of $0.3 \mathrm{GPa}$, there was almost negligible effect of hydrostatic pressures on the material response [33], [34]. To calibrate Kayenta for rate-dependence parameters, the experimental data were taken from Ref [35], where the plastic deformation and ductile fracture investigations were performed on Ti6Al4V for a variety of loading conditions (namely: tension, compression, shear) at different strain rates. In the present build of Kayenta, rate-dependence parameters, $\mathrm{T} 1$ and $\mathrm{T} 2$ are used to assign a value of the characteristic time according to the following formula

$$
\tau=f(\dot{\epsilon})=\mathrm{T}_{1}\left(\frac{1}{\dot{\epsilon}}\right) \mathrm{T}_{2}
$$

Taking the log of both sides, Eq. (1) becomes

$$
\log \tau=\log T_{1}-T_{2} \log \dot{\epsilon}
$$

Based on the unconfined compressive strength vs. strain rate data from Ref [35], a single-element test was first run by tuning the hardening parameters to match the quasistatic stress-strain curve. Then, the simulations were run at the strain rates provided in the experiment, and the characteristic time $\tau$ was tuned for the results to match the experimental stress-strain curves. These simulations were run using $\mathrm{T} 2=0$, thus providing the value of $\tau$ needed at each different strain rate. Figure 33 shows the plot of $\log \tau$ vs. $\log \mathrm{E}^{*}$, from which a linear fit then gave the values for T1 and T2 by fitting to Eq. (2). 


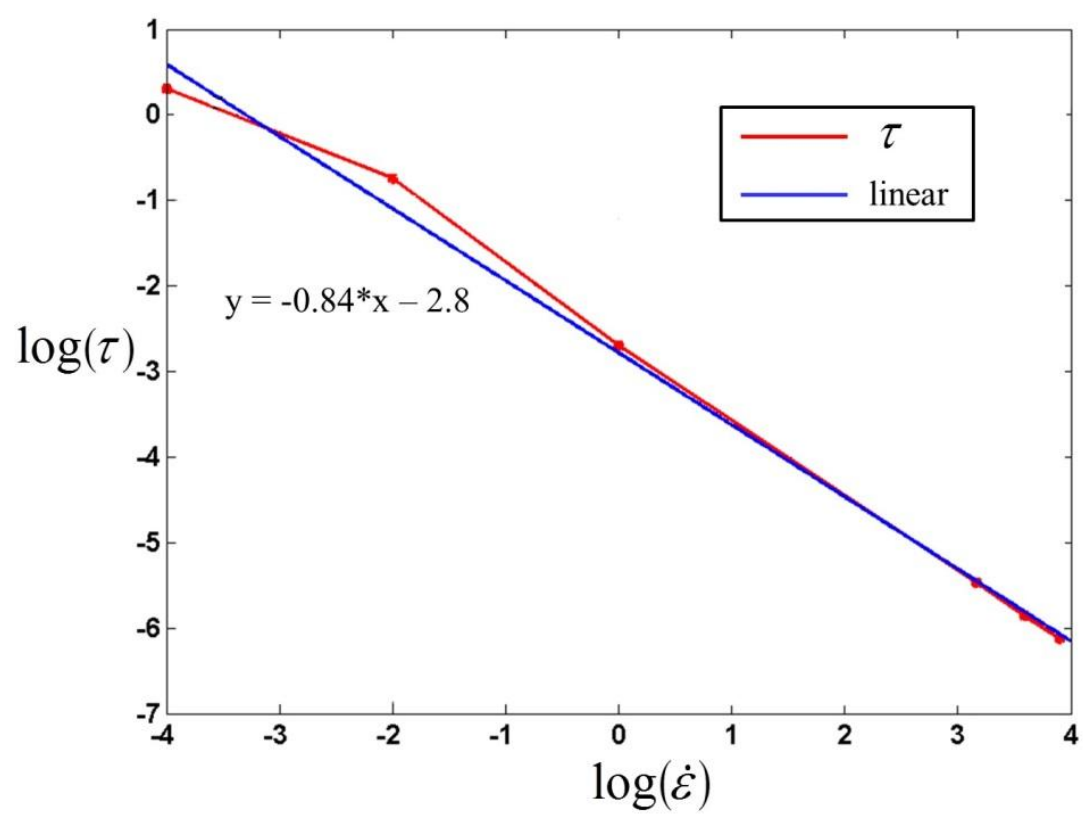

Figure 33: $\tau$ as a function of strain rate.

\subsection{Validation simulations using deterministic model}

After Kayenta was parameterized for rate dependence and hardening, deterministic simulations were run at the experimental speeds (i.e., $321 \mathrm{~m} / \mathrm{s}$ and $393 \mathrm{~m} / \mathrm{s}$ ) for two different Johnson-Cook failure parameter sets published in Ref [35], [27]. The pertinent input parameters for Kayenta are provided in Tables 3 through 5. Figures 34-35 show the color plot of damage where red is damaged and blue is intact material. At $321 \mathrm{~m} / \mathrm{s}$, the published parameter set 1 [35] was under-predicting damage, and published parameter set 2 [27] was over-predicting damage compared to the experiments. At $393 \mathrm{~m} / \mathrm{s}$, both failure parameter sets over-predicted damage. Also, the response was more ductile than the experiments. These simulations did not include the Johnson-Cook effect of temperature on the material strength.

Table 3: Input data used for Taylor-impact tests.

\begin{tabular}{|l|l|l|}
\hline Material Properties & Rate dependence & Hardening \\
\hline$\rho=4430 \mathrm{~kg} / \mathrm{m} 3$, & $\mathrm{T} 1=16 \times 10-4 \mathrm{~s}$ & $\mathrm{HC}=1 \mathrm{GPa}$, \\
$\mathrm{G}=44 \mathrm{GPa}$, & $\mathrm{T} 2=0.84$ & $\mathrm{RN}=-0.25 \mathrm{GPa}$ \\
$\mathrm{K}=91.7 \mathrm{GPa}$ & & \\
\hline
\end{tabular}

Table 4: Johnson-Cook failure model parameter sets.

\begin{tabular}{|l|l|l|l|l|l|}
\hline Johnson-Cook Failure model & D1 & D2 & D3 & D4 & D5 \\
\hline Hammer (Parameter set 1) & -0.81 & 1.18 & -0.15 & -0.012 & 2.10 \\
\hline Johnson (Parameter set 2) & -0.09 & 0.25 & -0.5 & -0.014 & 3.87 \\
\hline
\end{tabular}

Table 5: Mie-Grüneisen EOS inputs.

\begin{tabular}{|c|c|c|c|}
\hline $\begin{array}{c}\text { SNDSP0 } \\
\text { (Initial sound speed) } \\
\mathrm{m} / \mathrm{s}\end{array}$ & $\begin{array}{c}\mathrm{CV} \\
\text { (Specific heat) } \\
\mathrm{J} / \mathrm{kg} . \mathrm{C}\end{array}$ & $\gamma$ (Grüneisen parameter) & $\mathrm{S} 1$ \\
\hline 5130 & 570 & 1.23 & 1.28 \\
\hline
\end{tabular}




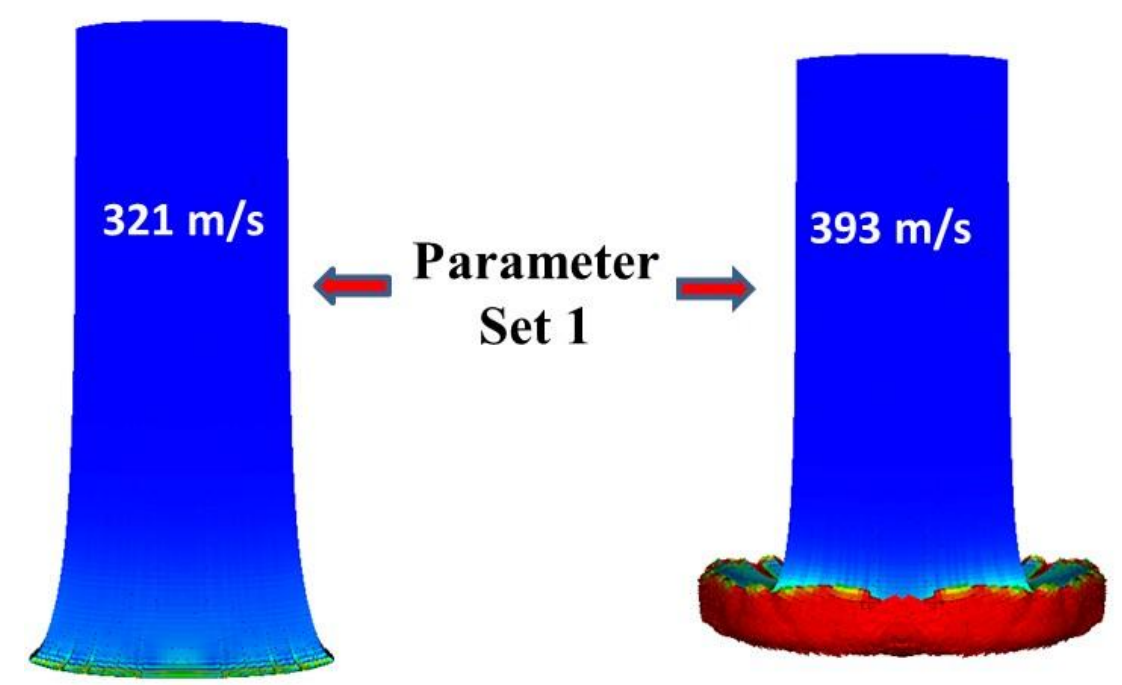

Figure 34: Plot of damage using Johnson-Cook failure model without thermal effects. Parameter set published by Hammer [35].

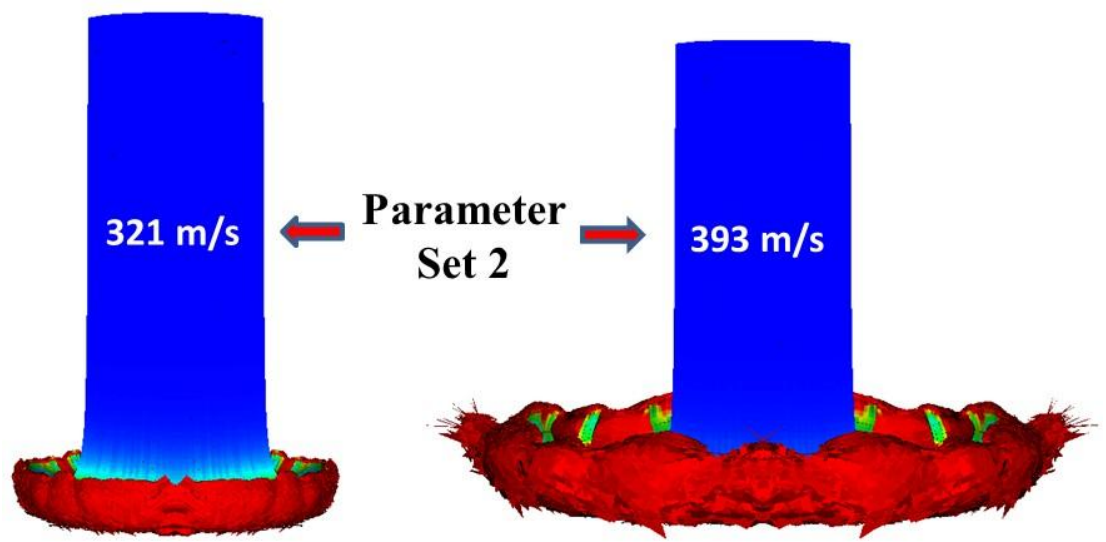

Figure 35: Plot of damage using Johnson-Cook failure model without thermal effects. Parameter set published by Johnson (cf. [27]).

At high rates, thermal effects are not negligible, and they need to be considered. An equation of state (EOS), which describes the pressure as a function of the density and temperature, characterizes the material response under such loading. Mie-Grüneisen is the most commonly used EOS, and the parameters for various metals are readily available in the literature. For Ti6Al4V, the EOS parameters were taken from Ref [36].

Figures 36-37 are the same simulations as Figures 34-35 except run with Mie-Grüneisen EOS. When using the EOS, the damage zones are considerably reduced using both the failure parameter sets.

Figure 38 shows the convergence study for the Taylor-anvil impact problem using a deterministic model. For all meshes, the snapshots of the deformation are taken at the same instant in time. Convergence issues persist with the damage zone echoing mesh texture and failing to converge with mesh refinement. Similar simulation shortcomings have been shown to be significantly alleviated for dynamic indentation problems by including realistic strength variability and scale effects [20], so a natural next step in systematic verification and validation is to investigate whether or not inclusion of such modeling features can likewise improve results for Taylor-anvil problems. 


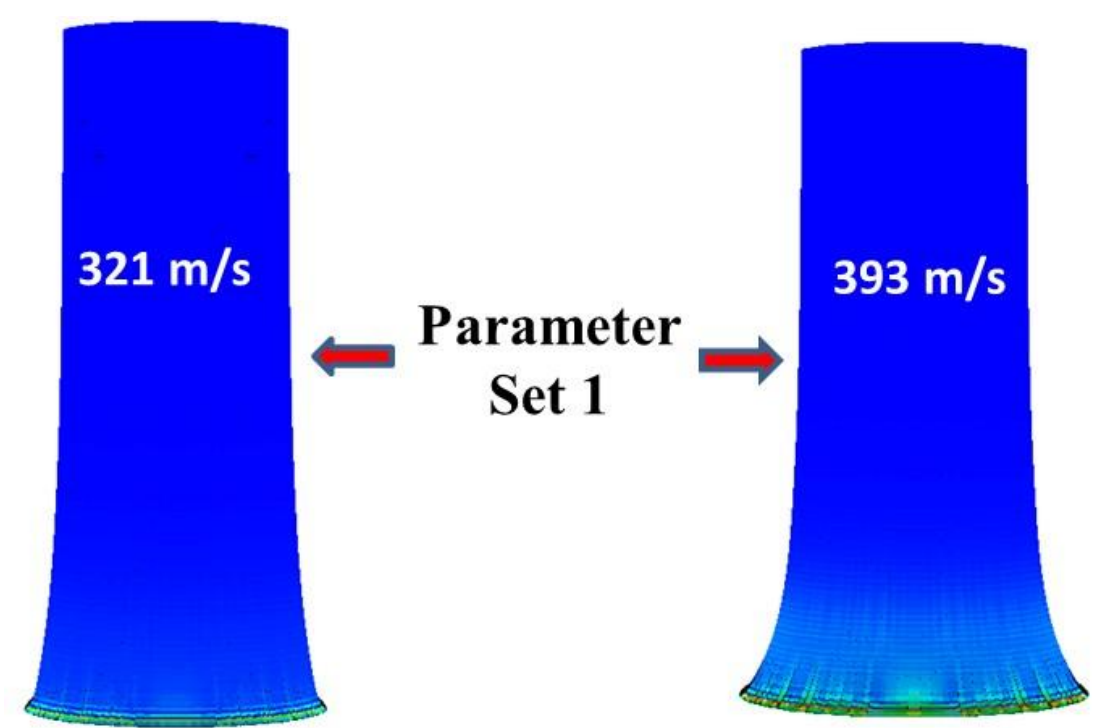

Figure 36: Plot of damage using Johnson-Cook failure model with thermal effects. Parameter set published by Hammer [35].

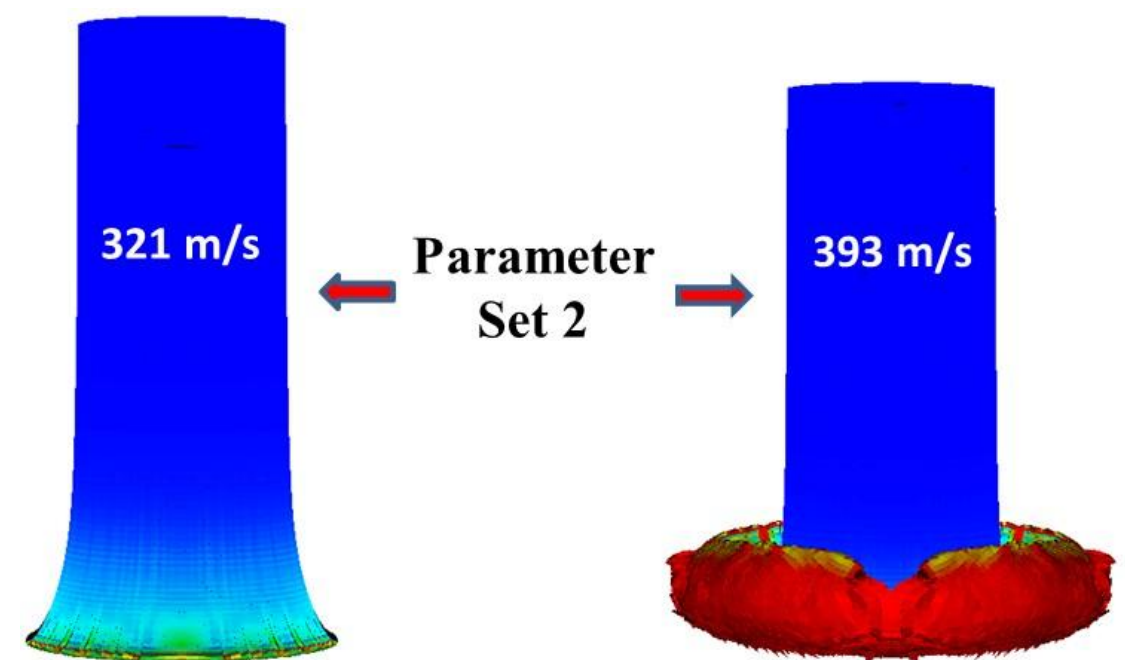

Figure 37: Plot of damage using Johnson-Cook failure model with thermal effects. Parameter set published by Johnson [1]. 

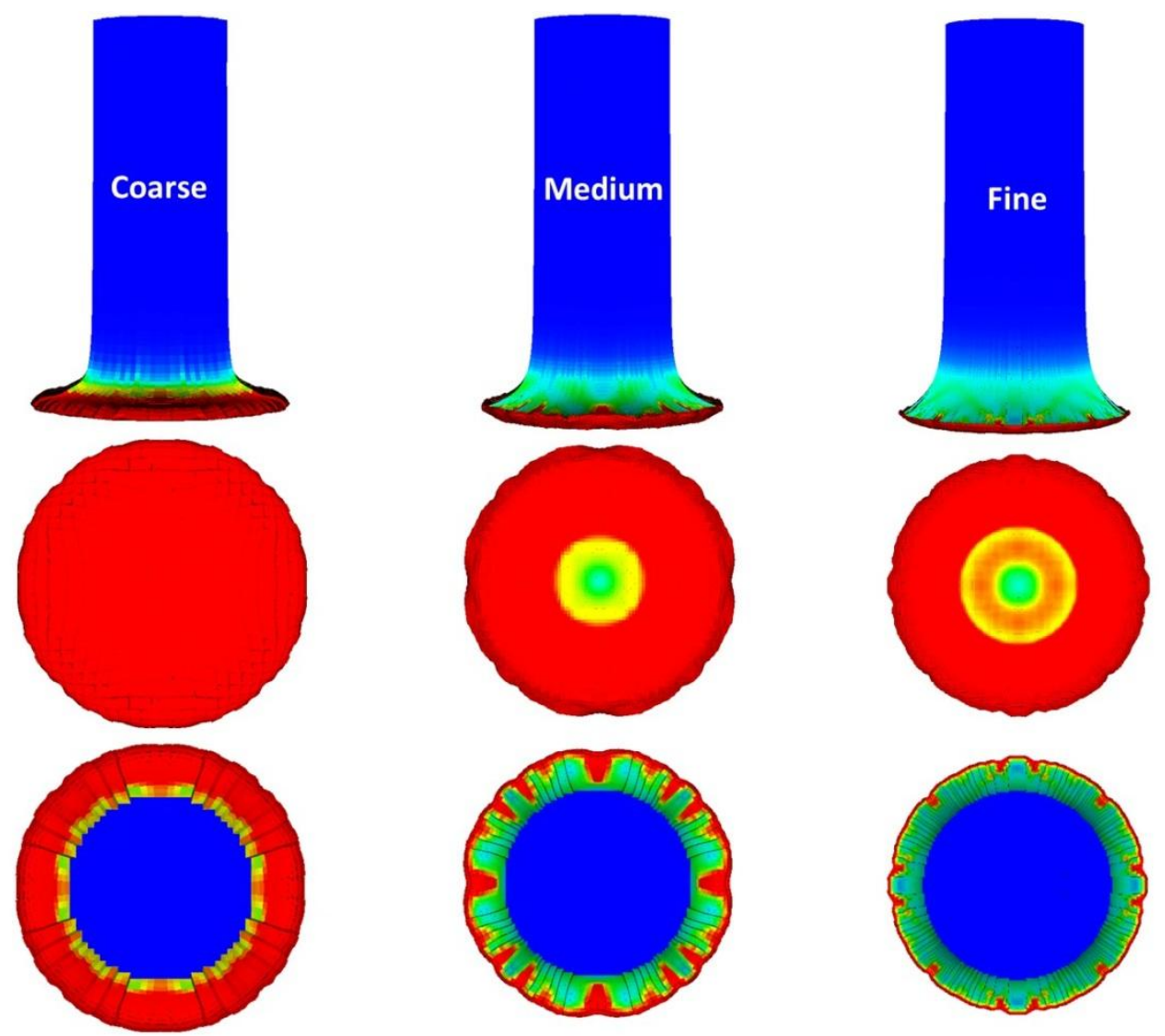

Figure 38: Severe mesh dependency for damage using a deterministic scale-insensitive model. Mesh resolution increases from left to right. Red is damaged and blue is intact material. Each column indicates the front view, the bottom view, and the top view of the damaged specimen respectively.

\subsection{Validation simulations using variability applied to rate-dependence parameters}

Failure parameters published by (cf. [27]) were used in this study. Previous work [20] statistically perturbed the quasistatic strength, but similar changes did not improve the convergence of the TaylorAnvil problem, at least not when using a strain-to-failure damage criterion. The previous work, however, used a time-to-failure damage criterion which also included scale effects. The current Taylor-anvil study instead used a strain-to-failure option which lacks the intrinsic rate effect of a time-to-failure option. To explore effects of variability on rate sensitivity, this Taylor-anvil study set variability of rate dependence parameters $\mathrm{T} 1$ and $\mathrm{T} 2$ so that low rates would show ductile response while high rates would (in principle) exhibit more brittle behavior. The T2 parameter controls the slope of the line in Figure 33, while T1 controls the intercept. An article has recently appeared in the literature [37] that uses microphysical arguments and dimensional analysis to identify a universal curve for rate dependence of strength for a wide range of brittle nonmetallic solids. For metals, a different article [38] also reports typical statistical variability of rate effects that is similar to observations for nonmetallics. To investigate if the universal curve from Ref [37] also applies to rate-dependence data for Ti6Al4V, an analysis was performed to best fit the data on the curve defined by

$$
\frac{\sigma_{\mathrm{f}}}{\sigma_{o}}=1+\left(\frac{\dot{\epsilon}}{\dot{\epsilon}_{o}}\right)^{\frac{2}{3}}
$$


where $\sigma_{0}$ and $\dot{\epsilon}_{0}$ are the characteristic stress and strain rates defined in terms of the material and the microstructural properties, both treated as fitting parameters. From the compression strain rate test data [35], a method of least squares was used to fit the model in Eq. (3). Figure 39 shows the least squares fit of the experimental data to the model with the characteristic stress and strain rates for the Ti6Al4V material, where the best-fit parameters for Ti6Al4V were found to be $\sigma_{0}=1495 \mathrm{MPa}$ and $\dot{\epsilon}=6.004 \times 10^{4} \mathrm{~s}^{-1}$.

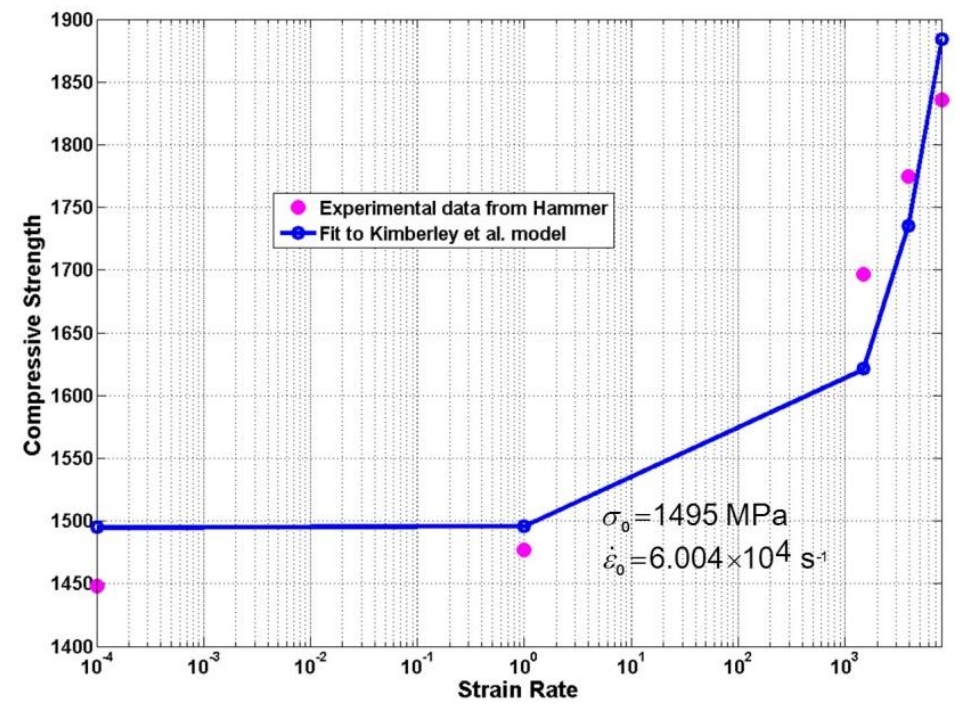

Figure 39: Least squares fit of Ti6Al4V experimental data [35] to Eq. (3).

This section explores the effect of statistically variable rate effects in the Duvaut-Lions component of the Kayenta model. Aside from material parameters, Taylor-impact simulations are known to be sensitive to the properties of the specimen-anvil contact surface. In the literature, choices for the friction coefficient $(\mu)$ are not always justified. Simulations presented in this section use the common choice, $\mu=0.1$ (cf. [15], [17]), but only because research on contact theories falls outside the scope of this study.

Figure 40 shows the comparison of damage with and without variability for the specimen speed of $393 \mathrm{~m} / \mathrm{s}$. No EOS was used in these simulations. A Weibull modulus (m) of 1 (meaning very high variability) was applied on T1. Figure 41 shows the same simulations as Figure 40, except that a Mie-Grüneisen EOS was also used. Snapshots are taken at the same instant in time for all simulations. The results using variability show an appropriate and realistic break in symmetry, while the deterministic simulations exhibit typical mesh texture bias. The level of damage and ductility observed when using a statistically variable $\mathrm{T} 1$ was not significantly different from the results in the deterministic simulations. In other words, despite this high level of variability in the T1 parameter, for the specimen speed of $393 \mathrm{~m} / \mathrm{s}$, noticeably brittle response was still not observed in the simulations. 

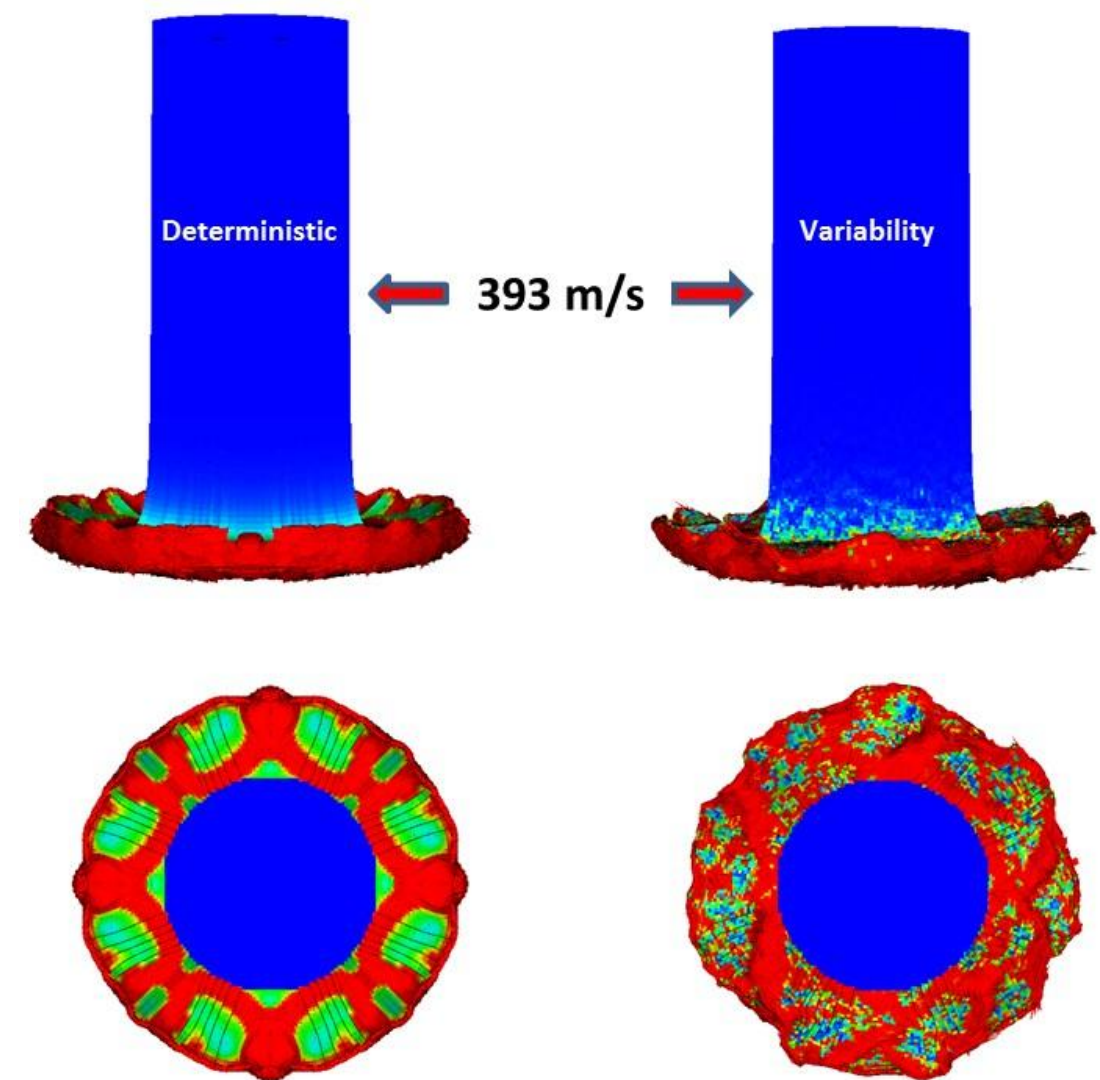

Figure 40: Sensitivity of rate-dependence parameter T1 on damage without EOS. The top row corresponds to the front view of the specimen and the bottom row corresponds to the top view of the specimen. Specimen speed is $393 \mathrm{~m} / \mathrm{s}$ and Weibull modulus $(\mathrm{m})$ is 1 . Red is damaged material, and blue is intact material. 


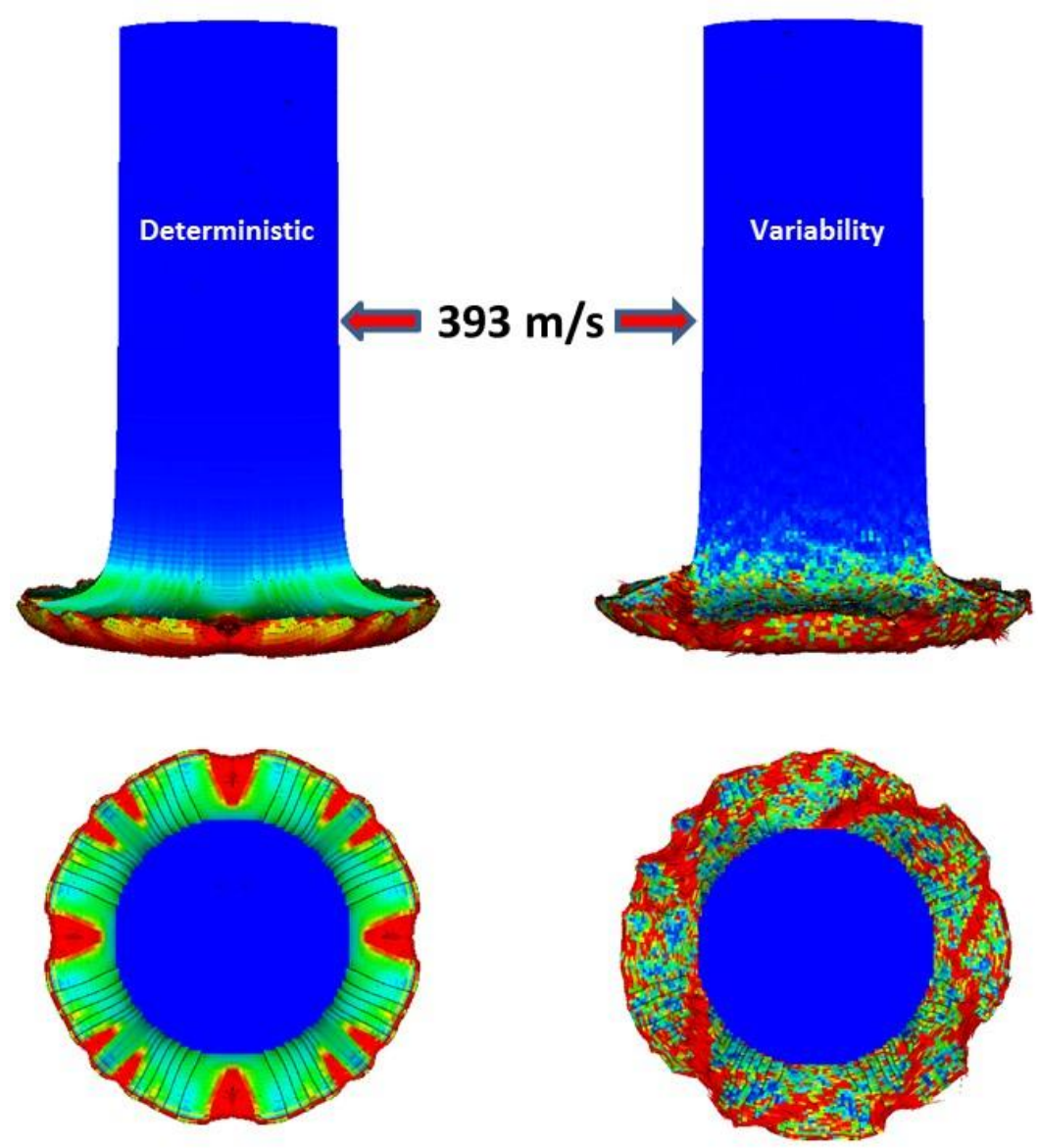

Figure 41: Sensitivity of rate-dependence parameter T1 on damage with EOS. The top row corresponds to the front view of the specimen and the bottom row corresponds to the top view of the specimen. Specimen speed is $393 \mathrm{~m} / \mathrm{s}$ and Weibull modulus $(\mathrm{m})$ is 1 . Red is damaged material, and blue is intact material.

A similar study was performed for the specimen speed of $321 \mathrm{~m} / \mathrm{s}$. With no EOS, the results were again similar to the results in the deterministic simulation, shown in Figure 42. With the inclusion of an EOS, Figure 43 shows the damage propagating at approximately $45^{\circ}$ which is a desired trait prior to fragmentation. However, at this speed, the numerical simulations did not predict fragments as observed in the experiment. Next, statistics were put on T2, and simulations with an EOS were run for a low Weibull modulus of 10 (moderately high variability in T2), a higher Weibull modulus of 20 (medium variability in T2), and a very high Weibull modulus of 30 (low variability in T2). 


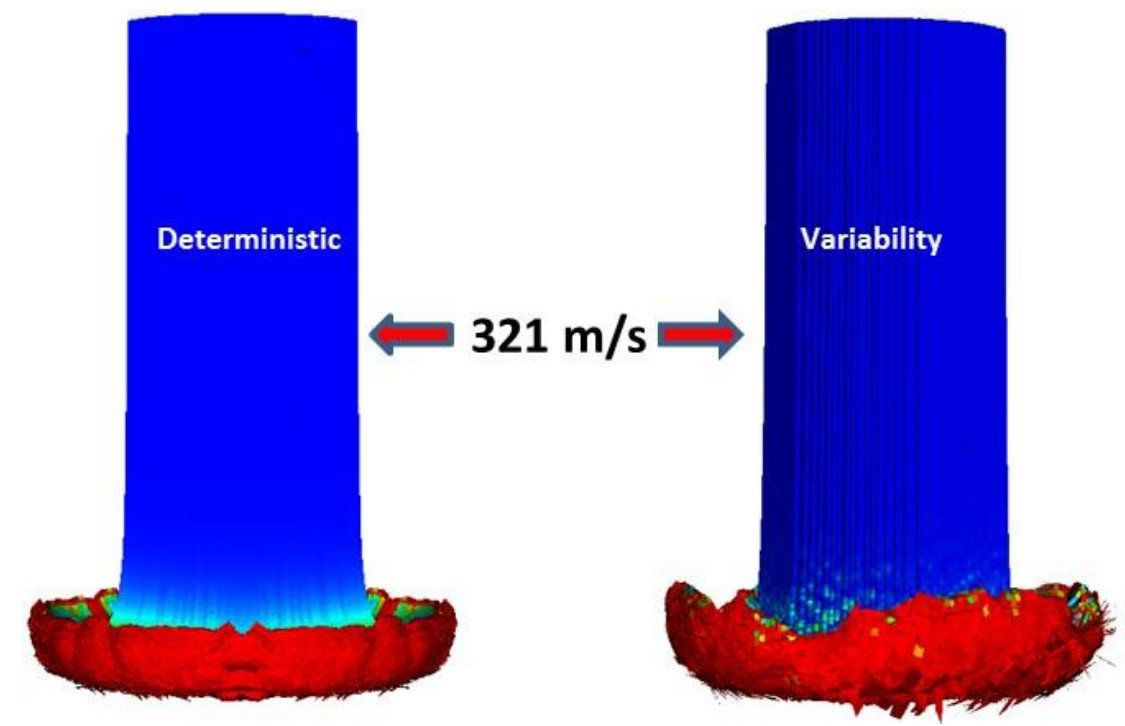

Figure 42: Sensitivity of rate-dependence parameter T1 on damage without EOS. Specimen speed is $321 \mathrm{~m} / \mathrm{s}$ and Weibull modulus (m) is 1 . Red is damaged material, and blue is intact material.

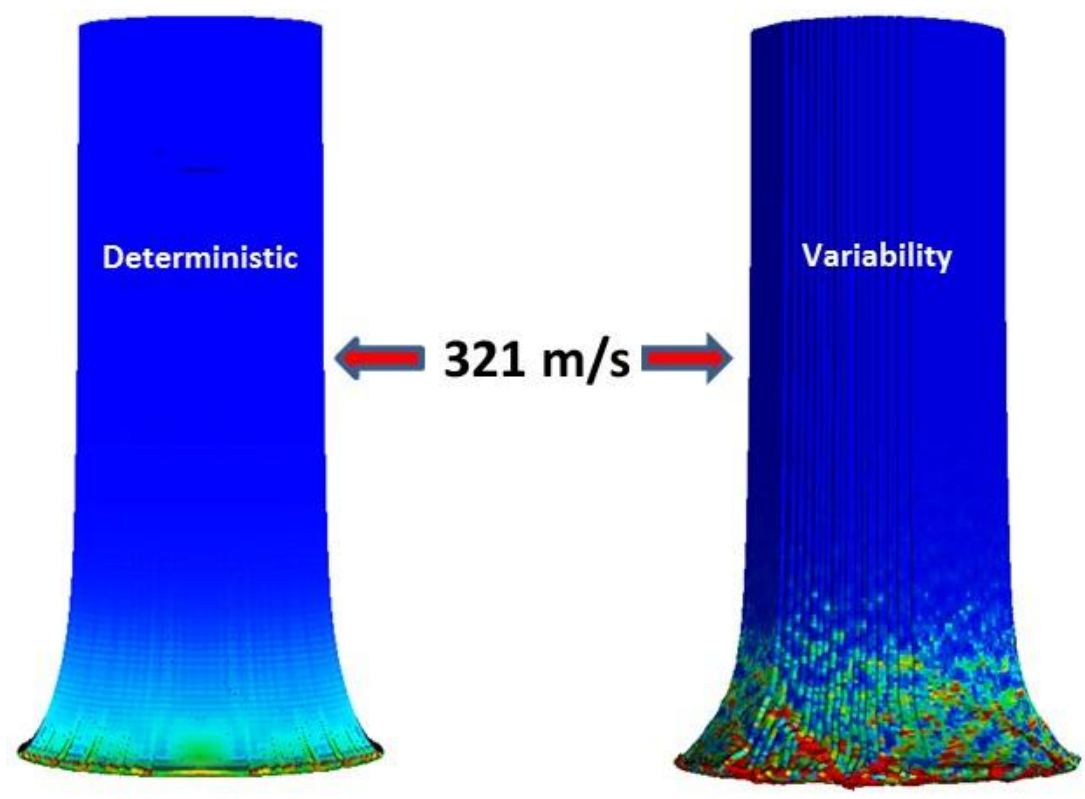

Figure 43: Sensitivity of rate-dependence parameter T1 on damage with EOS. Specimen speed is $321 \mathrm{~m} / \mathrm{s}$ and Weibull modulus (m) is 1 . Red is damaged material, and blue is intact material.

Figure 44 shows the snapshot of the simulations for different levels of variability in T2 (with no variability or scale effects in any other parameter). As expected, when the variability decreases, the results approach the deterministic model results. For a Duvaut-Lions model, a nonzero value of T1 provides a rate sensitive material response. A nonzero value of T2 (which is the exponent on strain rate) provides even greater rate sensitivity as the strain rate is increased. Since Taylor impact is a very highrate test, and since we are seeking ductile behavior at low rates, but brittle behavior at high rates, this observation suggests that the T2 parameter may play a significant role. A similar sensitivity study was performed on the simulations without an EOS for both specimen speeds to narrow down the best set of parameters that predict results closer to the experiments. 


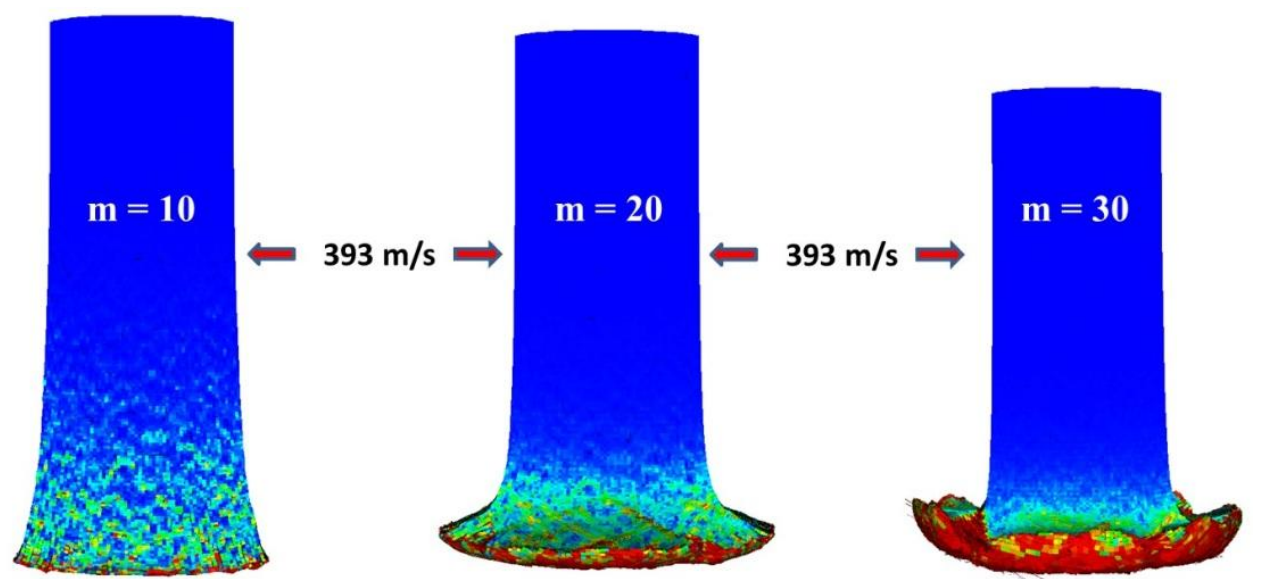

Figure 44: Color plot of damage with statistics put on T2. Blue is intact material and red is completely damaged material. The specimen impact speed was $393 \mathrm{~m} / \mathrm{s}$. The Weibull modulus $(\mathrm{m})$ increases from left to right i.e., variability decreases from left to right.

Figure 45 shows the results of the sensitivity study for the specimen speed of $321 \mathrm{~m} / \mathrm{s}$. For a higher Weibull modulus, the specimen was exhibiting localized damage, and for lower Weibull modulus, the bottom of the specimen was completely damaged. Also, the amount of ductility was decreased with the increase in variability.

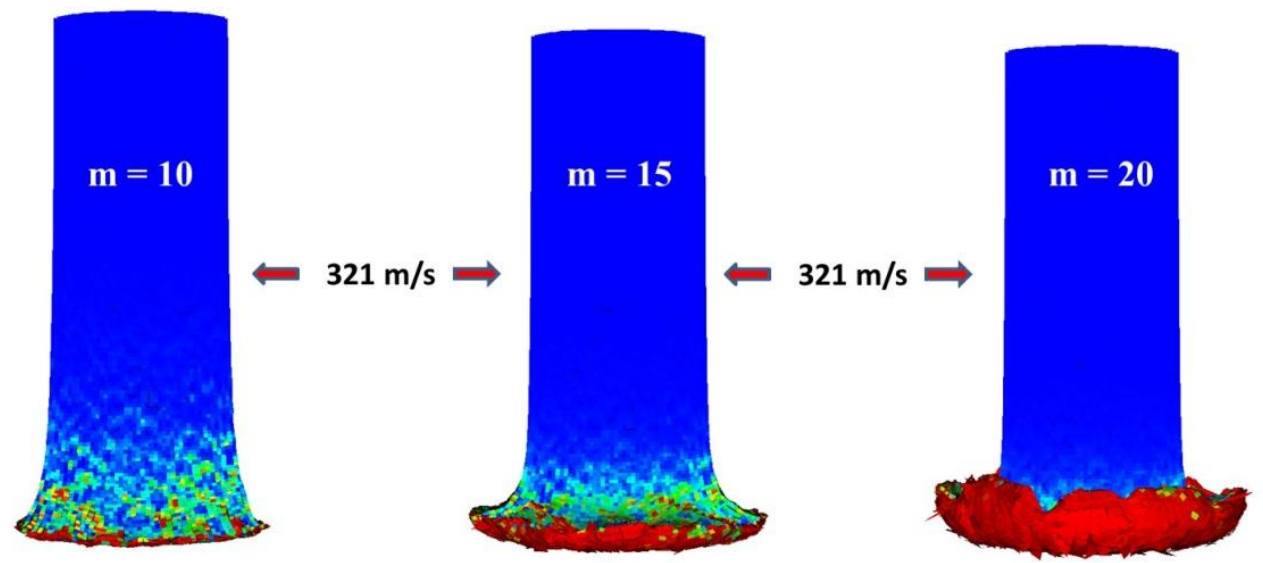

Figure 45: Color plot of damage with statistics put on T2. Blue is intact material and red is completely damaged material. Weibull modulus $(\mathrm{m})$ increases from left to right i.e., variability decreases from left to right.

The simulations presented so far used cylinder object that had stair-stepped descriptions of the angled boundaries. To investigate the associated errors, the simulations were re-run using a smooth cylinder object, where the particles are conformed with the boundary using the CPDI integrator. Figure 46 shows the same simulation as Figure 45, except run with a Weibull modulus (m) of 12. For the damage plot, red is damaged material, and blue is intact material. For the equivalent plastic strain plot, red is maximum equivalent plastic strain (value of 0.5 ), and blue is zero plastic strain. 


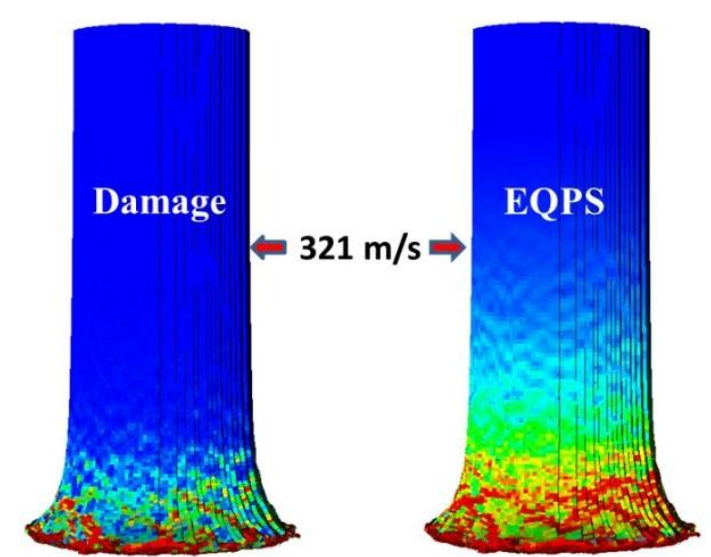

Figure 46: Color plot of damage and equivalent plastic strain with statistics put on T2. Weibull modulus $(\mathrm{m})$ is 12 .

Figure 47 shows the same results as Figure 46, except run with particles that are conformal to the boundary. The effect of smooth cylinder object on the damage patterns was negligible because all these simulations were run with considerable mesh refinement.

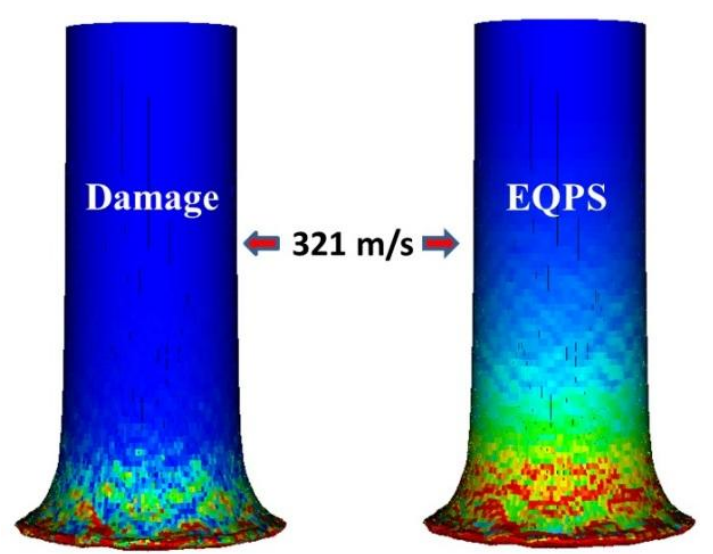

Figure 47: Same simulation as Figure 46 except run with particles conforming with the boundary.

Particle deletion has become a common approach to accommodate fragmentation in numerical simulations [17], [16], but its lack of a physical basis is why Kayenta's ability to induce loss of strength without particle deletion is preferred. The particle deletion technique was nevertheless explored in our framework to establish a basis of comparison against what is done in the literature [17], [16].

Figure 48 shows the damage plot with the specimen speed of $393 \mathrm{~m} / \mathrm{s}$ and no particle deletion (i.e., material separation is a pure constitutive response). Row A corresponds to simulation run with Weibull modulus (m) 30 and with the Mie-Grüneisen EOS. The top views of the specimen indicate several damaged zones oriented at $45^{\circ}$, which is desired (although these angled damage zones are not as large in size as evident in the experimental data). Rather than hiding the fully damaged particles in post-processing, the damaged material is shown in light gray. Row A of Figure 49 is the same as row B of Figure 48. For comparison, row B of Figure 49 corresponds to the same simulation as row A and at the same instant in time, except particle deletion was activated when the Kayenta's COHER value reached 0.005. It is evident that particle deletion gives different answers than without particle deletion, yet this technique is adopted in the literature to validate constitutive models. The comparison study in Figure 49 demonstrates that particle deletion is nonphysical and hence inappropriate for model validation. 


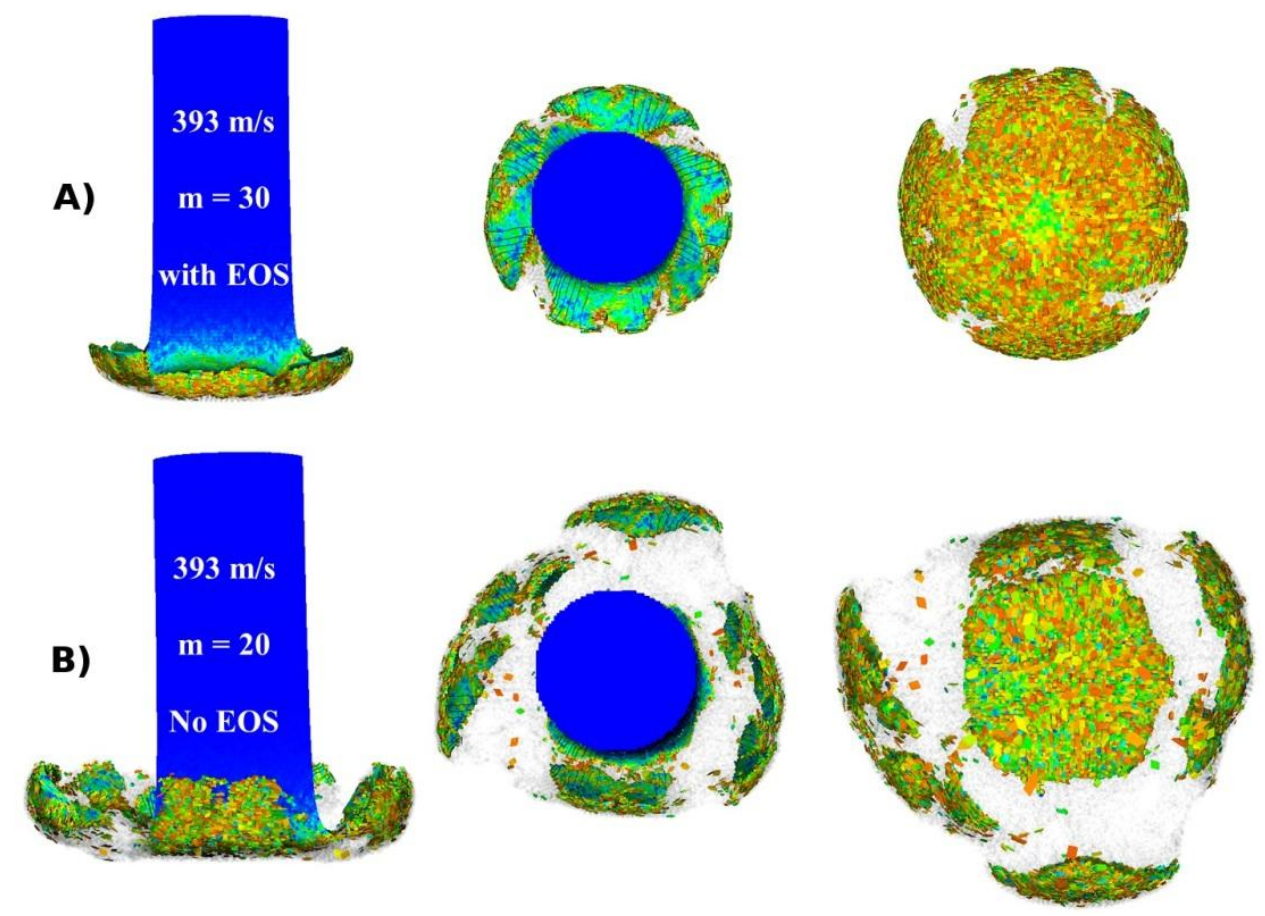

Figure 48: Results without particle deletion. A) Simulation with the Mie-Grüneisen EOS. Each row represents the front view, the top view, and the bottom view of the damaged specimen respectively. Damaged material is shown in gray. B) Corresponds to the same simulation as row A, except run without an EOS, and the Weibull modulus (m) is 20 to encourage discrete fragmentation. Impact speed is 393 $\mathrm{m} / \mathrm{s}$.

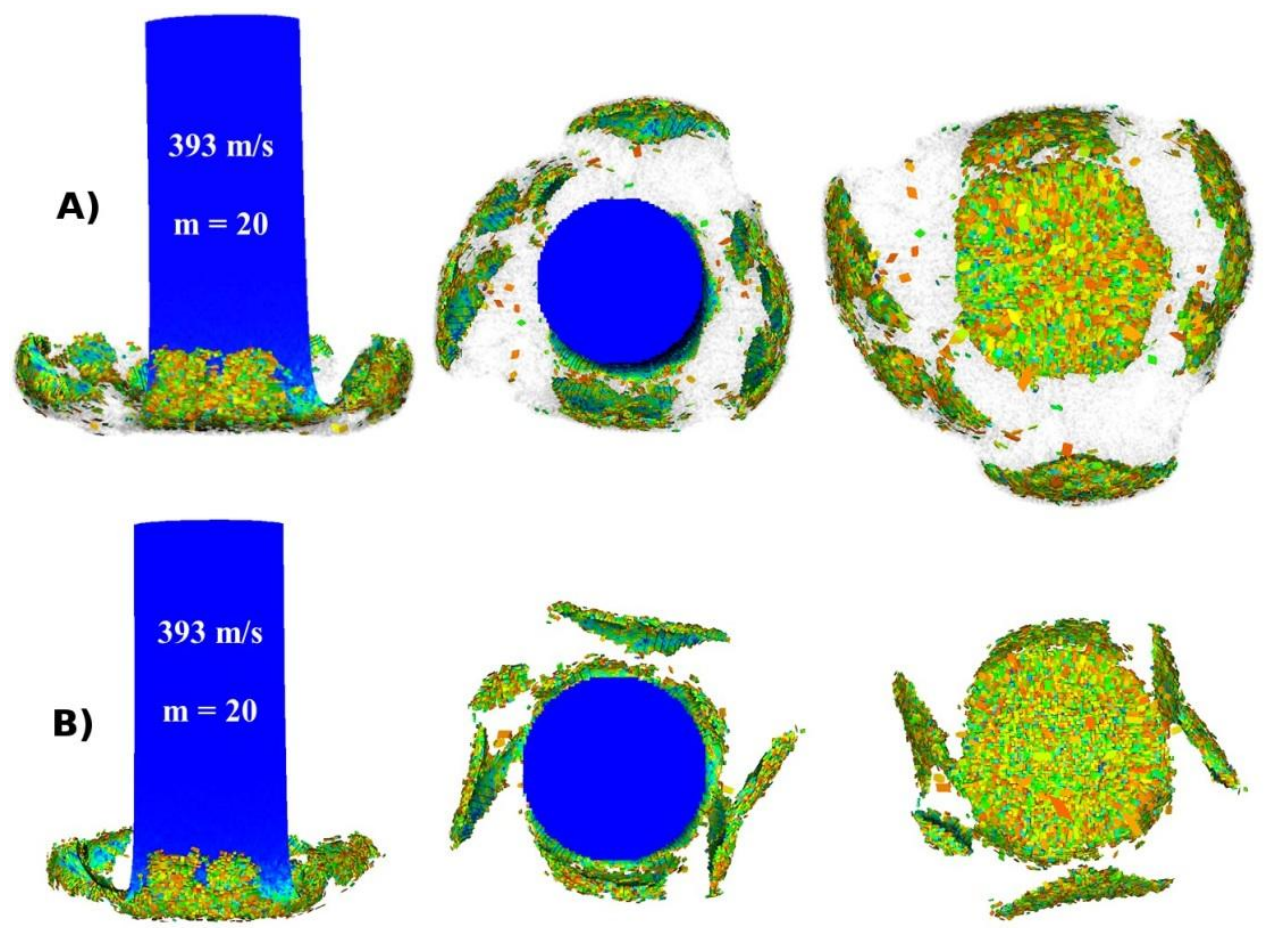

Figure 49: A) Simulation without the Mie-Grüneisen EOS. Each row represents the front view, the top view, and the bottom view of the damaged specimen respectively which used constitutive softening without particle deletion. B) Same simulation as row A except run with particle deletion.

To check if more localized damage zones and fragmentation can be predicted in the numerical simulations, variability was simultaneously applied to the melt temperature and to the Johnson-Cook failure parameter D5 (parameter associated with thermal softening). Figure 50 shows the damage for the 
best prediction achieved so far in the numerical study. The effect of putting variability simultaneously on the melt temperature and the Johnson-Cook failure parameter D5 had almost negligible effect on the damage patterns.

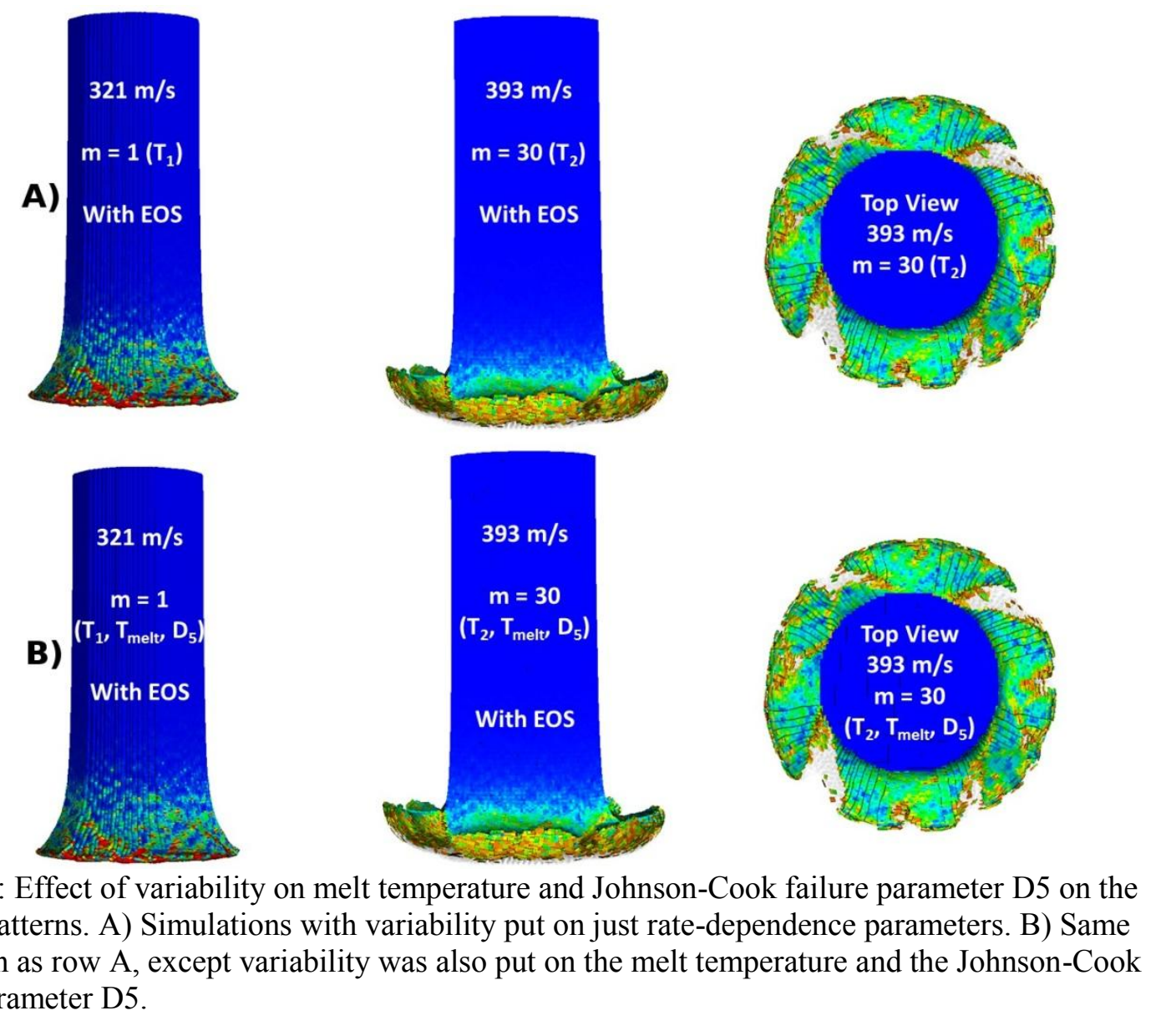

\subsection{Kinematic locking in Taylor-anvil impact test}

Like conventional finite-element methods, the material point method can potentially exhibit anomalous (i.e., nonphysical) kinematic locking because of the use of linear shape functions on a rectangular grid. The nonphysical checker-boarding of the vertical component of stress seen in row A of Figure 51 is because of the locking issue. This locking is alleviated by implementing standard antilocking algorithms [39]. Row B corresponds to the same simulation as row A except run with one of these algorithms, called pressure stabilization. Though the checker-boarding is alleviated, the effect of pressure stabilization on the damage patterns is negligible. 

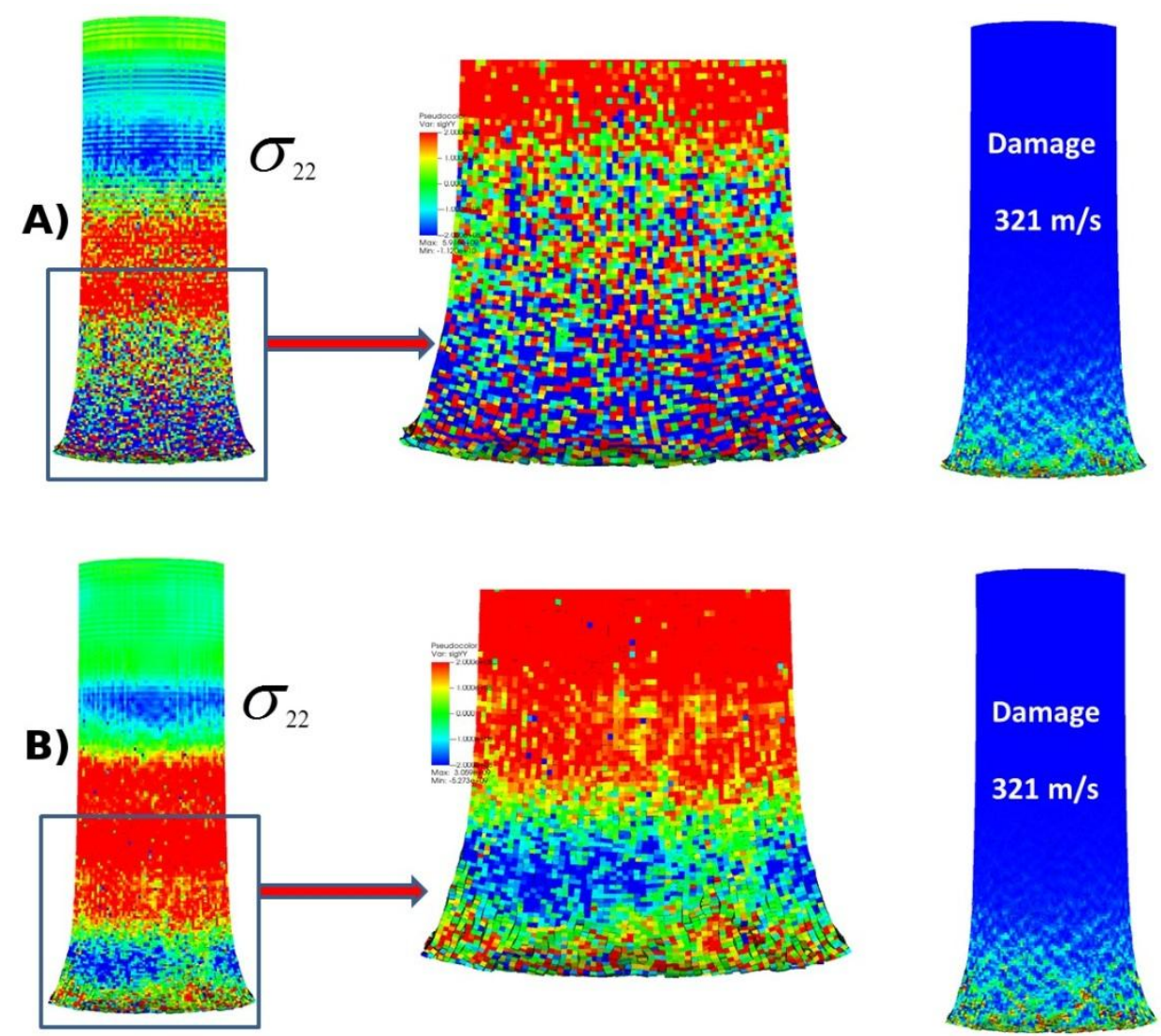

Figure 51: Effect of pressure stabilization on the damage patterns. A) Simulation without pressure stabilization technique. Color plot of $\sigma 22$ where the checker-boarding is the symptom of locking. B) Same simulation as row A, except run with pressure stabilization

\section{Conclusions}

For the unnotched Charpy test, the energy absorbed by the specimen, the bend angle of the specimen and the damage patterns using Kayenta's various failure options that include aleatory uncertainty and scale effects were compared against the experiments. Initially, the simulations were run at 10 times the experimental striker speeds to make the simulations complete faster and also to investigate the rate effects on the specimen damage patterns. The average bend angle was $7.7 \%$ higher than the experimental observation, and the average amount of energy absorbed was $24 \%$ higher. Not surprisingly, when the simulations were run at the same speeds as the experiments, the results gave much better agreement with the data: the average bend angle was $0.4 \%$ higher than observed in the experiment, and the average amount of energy absorbed was $1.2 \%$ lower. However, mesh refinement is required to see if the results converge. The fracture patterns did not converge using only statistical variability in strength, but results improved when using both statistical variability in strength and scale effects to give damage patterns concentrated at the bottom-center of the specimen. For the amount of energy absorbed by the specimen, statistical variability in strength and scale effects using strain-to-failure softening option did not eliminate convergence issues during the softening phase. Therefore, it is prudent to investigate if scale-dependent time-to-failure softening option with statistical variability in strength and scale effects could mitigate the convergence issue during softening.

For the Taylor-anvil impact test, the damage patterns using spatially variable rate-dependence parameters were compared against the experiments. Since rate dependence was never used simultaneously with softening in Kayenta before, a systematic verification study was presented. First, mesh sensitivity using a deterministic model was demonstrated, then variability was applied to the ratedependence parameters $\mathrm{T} 1$ and $\mathrm{T} 2$. Though improved results were demonstrated, the results still do not adequately capture the experimental observations. At the specimen speed of $321 \mathrm{~m} / \mathrm{s}$, the simulations 
with statistics put on $\mathrm{T} 1$ with EOS were predicting results closer to the experiments. In these simulations, though the damage was propagating at $45^{\circ}$, that did not induce fragments in the specimen as observed in the experiment. At higher speeds of $393 \mathrm{~m} / \mathrm{s}$, fragmentation was observed, but the fragments were not as large in size as evident in the experimental data.

In the numerical study presented for Taylor-anvil impact test, strength was taken to be pressure independent based on the available experimental data (up to confining pressures of $0.3 \mathrm{GPa}$ ). However, the actual impact pressures reached in the numerical simulations fall well outside the experimental range. Therefore, it is recommended to run the simulations with pressure-dependent strength to explore the sensitivity of damage patterns to this feature.

\section{Acknowledgements}

The authors gratefully acknowledge the faithful support of the US Army Technology Programs Office, PD Main Battle Tank Systems, without which this effort would not have been possible.

\section{References}

[1] G. R. Johnson and W. H. Cook, "Fracture Characteristics of Three Metals Subjected to Various Strains, Strain Rates, Temperatures and Pressures," Engineering Fracture Mechanics, vol. 21, no. 1, pp. 31-48, 1985.

[2] M. A. Meyers, Dynamic Behavior of Materials, NJ: John Wiley \& Sons, Inc., 1994.

[3] R. Boyer and G. Welsch, Materials Properties Handbook: Titanium Alloys, 1994.

[4] A. L. Gurson, "Continuum Theory of Ductile Rupture by Void Nucleation and Growth: Part IYield Criteria and Flow Rules for Porous Ductile Media," Journal of Engineering Materials and Technology, vol. 99, no. 1, pp. 2-15, 1977.

[5] F. A. McClintock, "A Criterion for Ductile Fracture by the Growth of Holes," Journal of Applied Mechanics, vol. 35, no. 2, pp. 363-371, 1968.

[6] J. R. Rice and D. M. Tracey, "On the Ductile Enlargement of Voids in Triaxial Stress Fields," $J$. Mech. Phys. Solids, vol. 17, pp. 201-217, 1969.

[7] M. L. Wilkins, R. D. Streit and J. E. Reaugh, Cumulative-strain-damage model of ductile fracture: simulation and prediction of engineering fracture tests, Livermore, CA: Lawrence Livermore National Laboratory, 1980.

[8] Y. Bai and T. Wierzbicki, "A new model of metal plasticity and fracture with pressure and Lode dependence," International Journal of Plasticity, pp. 1071-1096, 2008.

[9] A. G. Charpy, "Note sur L'essai des métaux á la flexion par choc de barreaux entaillés. Association internationale pour l'essai des matériaux.," in This paper was published in Soc. Ing. Civ. de Francis, June 1901.pp. 848-877., Congrés de Budapest., 1901.

[10] ASTM E23, Standard Test Methods for Notched Bar Impact Testing of Metallic Materials, 2007.

[11] G. I. Taylor, "The Use of Flat-Ended Projectiles for Determining Dynamic Yield Stress," Proceedings of the Royal Society, pp. 194-298, 1948.

[12] X. Xiao, W. Zhang, G. Wei and Z. Mu, "Effect of projectile hardness on deformation and fracture behavior in the Taylor impact test," Materials \& Design, pp. 4913-4920, December 2010.

[13] P. J. Maudlin, J. F. Bingert and G. T. Gray III, "Low-symmetry plastic deformation in BCC tantalum: experimental observations, modeling and simulations," International Journal of Plasticity, vol. 19, pp. 483-515, 2003.

[14] S. S. Gautam, R. Babu and P. M. Dixit, "Ductile fracture simulation in the Taylor rod impact test using continuum damage mechanics," International Journal of Damage Mechanics, vol. 20, pp. 347369,2010 . 
[15] X. Teng, T. Wierzbicki, S. Hiermaier and I. Rohr, "Numerical prediction of fracture in the Taylor test," International Journal of Solids and Structures, pp. 2929-2948, 2005.

[16] X. Xiao, W. Zhang, G. Wei, Z. Mu and Z. Guo, "Experimental and numerical investigation on the deformation and failure behavior in the taylor test," Materials \& Design, pp. 2663-2674, 2011.

[17] K. G. Rakvåg, T. Børvik and O. S. Hopperstad, "A numerical study on the deformation and fracture modes of steel projectiles during Taylor bar impact tests," International Journal of Solids and Structures, vol. 51, no. 3-4, pp. 808-821, 2014.

[18] J. Guilkey, T. Harman, J. Luitjens, J. Schmidt, J. Thornock, J. de St. Germain, S. Shankar, J. Peterson and C. Brownlee, "Uintah User Guide Version 1.1," SCI Institute Technical Report, University of Utah, 2009.

[19] R. M. Brannon, A. F. Fossum and O. E. Strack, "KAYENTA : theory and user's guide," Sandia National Laboratories, 2009.

[20] R. M. Brannon, J. M. Wells and O. E. Strack, "Validating Theories for Brittle Damage," Metallurgical and Materials Transactions A, 2007.

[21] O. E. Strack, R. B. Leavy and R. M. Brannon, "Aleatory uncertainty and scale effects in computational damage models for failure and fragmentation," International Journal for Numerical Methods in Engineering, 2014.

[22] L. Graham-Brady, "Statistical characterization of meso-scale uniaxial compressive strength in brittle materials with randomly occurring flaws," International Journal of Solids and Structures, vol. 47, no. 18-19, pp. 2398-2413, 2010.

[23] M. Timmel, S. Kolling, P. Osterrieder and P. A. Du Bois , "A finite element model for impact simulation with laminated glass," International Journal of Impact Engineering, pp. 1465-1478, 2007.

[24] R. M. Brannon and S. Leelavanichkul, "Survey of Four Damage Models for Concrete," Sandia National Laboratories, 2009.

[25] J. M. Lacy, J. K. Shelley, J. H. Weathersby, G. S. Daehn, J. Johnson and G. Taber, "OptimizationBased Constitutive Parameter Identification from Sparse Taylor Cylinder Data," SAVIAC 81st Shock and Vibration Symposium, Orlando, FL, Oct. 24-28, 2010.

[26] G. Duvaut and J. Lions, "Les Inéquations en Mécanique et en Physique," Paris, 1972.

[27] D. R. Lesuer, "Experimental Investigationsof Material Models for Ti-6Al-4V Titanium and 2024-T3 Aluminum," DOT/FAA/AR-00/25, 2000.

[28] E. El-Magd and M. Abouridouane, "Characterization, modelling and simulation of deformation and fracture behaviour of the light-weight wrought alloys under high strain rate loading," International Journal of Impact Engineering, vol. 32, no. 5, pp. 741-758, 2006.

[29] A. S. Khan, R. Kazmi and B. Farrokh, "Multiaxial and non-proportional loading responses, anisotropy and modeling of Ti-6Al-4V titanium alloy over wide ranges of strain rates and temperatures," International Journal of Plasticity, vol. 23, no. 6, pp. 931-950, 2007.

[30] M. Martin, T. Shen and N. Thadhani, "Instrumented anvil-on-rod impact experiments for validating constitutive strength model for simulating transient dynamic deformation response of metals," Materials Science and Engineering, no. 494, pp. 416-424, 2008.

[31] W. A. Spitzig, R. J. Sober and O. Richmond, "Pressure Dependence of Yielding and Associated Volume Expansion in Tempered Martensite," Acta Metallurgica, vol. 23, no. 7, pp. 885-893, July 1975.

[32] W. A. Spitzig, R. J. Sober and O. Richmond, "The Effect of Hydrostatic Pressure on the Deformation Behavior of Maraging and HY-80 Steels and its Implications for Plasticity Theory," Metallurgical Transactions, vol. 7, no. 11, pp. 1703-1710, 1976.

[33] E. Hanina, D. Rittel and Z. Rosenburg, Pressure sensitivity of adiabatic shear banding in metals, Haifa, Israel: AIP Applied Physics Letters, 2007. 
[34] A. S. Khan, "Response \& Modeling Of Electron Beam Single Melt Ti-6Al-4V Alloys," U.S. Army Research Office, 2010.

[35] J. T. Hammer, Plastic Deformation and Ductile Fracture of Ti-6Al-4V under Various Loading Conditions, The Ohio State University, 2012.

[36] D. J. Steinberg, "Equation of State and Strength Properties of Selected Materials," Lawrence Livermore National Laboratory, Livermore, CA, 1996.

[37] J. Kimberley, K. Ramesh and N. Daphalapurkar, "A scaling law for the dynamic strength of brittle solids," Acta Materialia, vol. 61, no. 9, pp. 3509-3521, 2013.

[38] D. Casem and H. Meyer, "Re-examining the Taylor Impact Test Using a Finite-Element-Based Inverse Problem Methodology," in SEM Annual Conference \& Exposition on Experimental and Applied Mechanics , 2005.

[39] C. Mast, P. Mackenzie-Helnwein, P. Arduino, G. Miller and W. Shin, "Mitigating kinematic locking in the Material Point Method," Journal of Computational Physics, vol. 231, no. 16, pp. 5351-5373, 2012.

[40] G. R. Johnson and T. J. Holmquist, "An improved computational constitutive model for brittle materials," in AIP Conf. Proc. 309, Colorado Springs, CO, 1994. 